\title{
Probing electroweak baryogenesis induced by extra bottom Yukawa coupling via EDMs and collider signatures
}

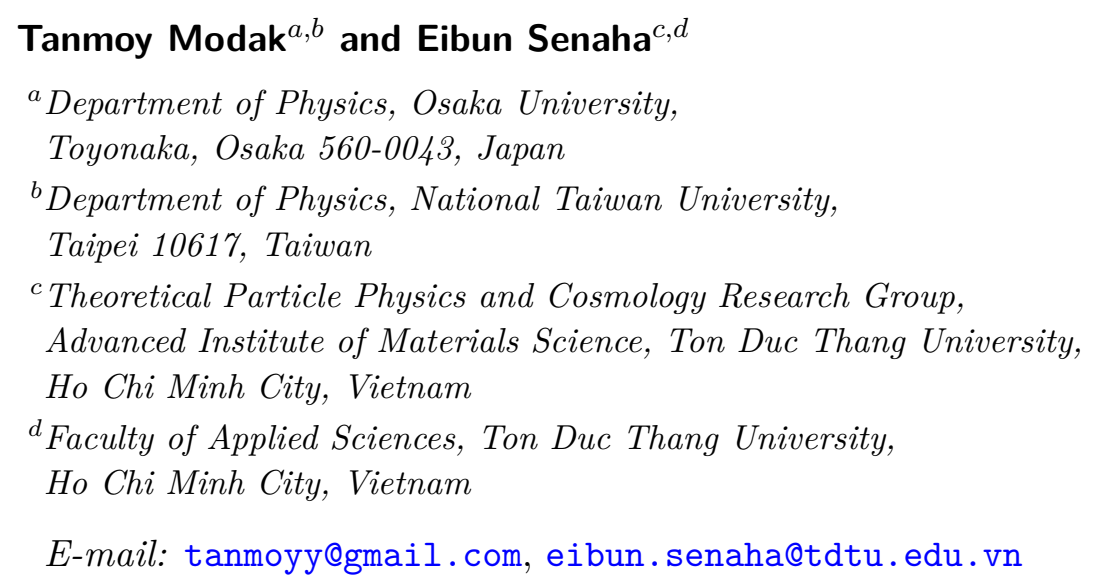

ABSTRACT: We study the prospect of probing electroweak baryogenesis driven by an extra bottom Yukawa coupling $\rho_{b b}$ in a general two Higgs doublet model via electric dipole moment (EDM) measurements and at the collider experiments. The parameter space receives meaningful constraints from $125 \mathrm{GeV}$ Higgs $h$ boson signal strength measurements as well as several heavy Higgs boson searches at the Large Hadron Collider (LHC). In addition, we show that the asymmetry of the CP asymmetry of inclusive $B \rightarrow X_{s} \gamma$ decay would provide complementary probe. A discovery is possible at the LHC via $b g \rightarrow b A \rightarrow$ $b Z h$ process if $\left|\rho_{b b}\right| \sim 0.15$ and $250 \mathrm{GeV} \lesssim m_{A} \lesssim 350 \mathrm{GeV}$, where $A$ is CP odd scalar. For $m_{A}>2 m_{t}$ threshold, where $m_{t}$ is the top quark mass, one may also discover $b g \rightarrow b A \rightarrow b t \bar{t}$ at the high luminosity LHC run if an extra top Yukawa coupling $\left|\rho_{t t}\right| \sim 0.5$, though it may suffer from systematic uncertainties. For completeness we study $g g \rightarrow t \bar{t} A \rightarrow t \bar{t} b \bar{b}$ but find it not promising.

Keywords: Beyond Standard Model, CP violation, Heavy Quark Physics, Higgs Physics ARXIV EPRINT: 2005.09928 


\section{Contents}

1 Introduction 1

2 Framework 3

3 Parameter space 5

3.1 Flavor constraints

3.2 EDMs 9

3.3 Direct search limits 14

4 Collider signatures $\quad 16$

4.1 The $b g \rightarrow b A \rightarrow b Z h$ process 16

$\begin{array}{lll}4.2 & \text { The } b g \rightarrow b A \rightarrow b t \bar{t} \text { process } & 19\end{array}$

4.3 The $g g \rightarrow t \bar{t} A \rightarrow t \bar{t} b \bar{b}$ process 22

$5 \quad$ Discussion and summary $\quad 23$

$\begin{array}{ll}\text { A EDMs } & 25\end{array}$

\section{Introduction}

The existence of the matter-antimatter asymmetry is unswervingly established over the years by various cosmological observations such as the cosmic microwave background anisotropies and big-bang nucleosynthesis [1]. It has been understood that the Universe started with equal number of baryons and antibaryons, but later evolved into baryon dominated Universe dynamically via a mechanism called baryogenesis. A successful baryogenesis requires three necessary conditions namely, baryon number violation, charge conjugation (C) and charge conjugation-parity (CP) violation and, departure from thermal equilibrium, laid out by Sakharov in 1967 [2]. A plethora of baryogenesis scenarios have been proposed so far to account for the observed baryon asymmetry of the Universe (BAU), however, its origin is still unclear. After the discovery of the $125 \mathrm{GeV}$ Higgs boson at the Large Hadron Collider (LHC) [3], a significant attention has been directed in particular to electroweak baryogenesis (EWBG) [4, 15-89] $]^{1}$ mechanism for its direct connections to Higgs physics and, its testability at the ongoing experiments. The Standard Model (SM) belongs to this class, however the $\mathrm{CP}$ violation is too small and, the electroweak symmetry breaking is not strongly first order phase transition (EWPT) to drive departure from thermal equilibrium.

While we do not have any strong experimental evidence of new physics yet, multiHiggs sector is the natural consequence of most ultraviolet (UV) theories due to enlarged

\footnotetext{
${ }^{1}$ For some reviews see e.g. refs. [5-14].
} 
symmetries. Whatever the fundamental theory might be, their effective descriptions at $\mathcal{O}(100) \mathrm{GeV}$ scale should resemble the $\mathrm{SM}$ in light of the latest experimental results. As for the Higgs sector, two cases are conceivable: one is that all the new scalars are much heavier than $\mathcal{O}(100) \mathrm{GeV}$ scale, thereby the Higgs sector is effectively reduced to the SM, while the other is that new scalars have $\mathcal{O}(100) \mathrm{GeV}$ masses but their couplings to the gauge bosons and fermions are SM like, mimicking the SM. From the viewpoint of new physics discovery potential, it is timely to consider the latter case and investigate whether the aforementioned cosmological issue can be solved or not. Since we have already confirmed the existence of the Higgs doublet in nature, it is tempting to us to think of additional Higgs doublets in analogy with the fact that all the fermions come in three copies.

The general two Higgs doublet model (g2HDM) is one of the simplest renormalizable low-energy models where the scalar sector of the SM is extended by an extra scalar doublet. ${ }^{2}$ Without the presence of discrete symmetry, in g2HDM, both the scalar doublets couple with up- and down-type fermions at tree level. In the mass eigenbasis of the fermions $(F)$, one has two independent Yukawa couplings $\lambda_{i j}^{F}$ and $\rho_{i j}^{F}$, where the former is real and diagonal that are responsible for the fermion mass generation, while the latter is complex and non-diagonal. Such complex couplings can provide additional CP violating sources beyond the usual Cabibbo-Kobayashi-Maskawa (CKM) framework [92] of the SM.

EWBG in g2HDM is widely investigated in refs. [79-86]. This model can simultaneously accommodate the strong first-order EWPT and sufficient amount of CP violation which the SM fails to provide. The most natural EWBG scenario in g2HDM would be the case in which BAU is driven by the extra top Yukawa coupling $\left(\rho_{t t}\right)$ of $\mathcal{O}(0.01-1)$ in magnitude with moderate size of the CP phase $\left(\rho_{t t}\right.$-EWBG) [84]. The devoted collider study of this scenario is conducted in ref. [93].

As a complementary study to $\rho_{t t}$-EWBG, the present authors consider a scenario in which the CP phase of $\rho_{t t}$ is approximately zero and the extra bottom Yukawa coupling $\left(\rho_{b b}\right)$ plays a dominant role in generating BAU $\left(\rho_{b b}\right.$-EWBG) [85]. It is demonstrated that BAU can reach the observed level if $\left|\operatorname{Im} \rho_{b b}\right| \gtrsim 0.058$ with generous assumptions on a Higgs bubble wall profile. There exist several direct and indirect search constraints on the parameter space for $\rho_{b b}$-EWBG such as $h$ boson signal strength measurements, heavy Higgs searches at the LHC. The $\rho_{b b}$-EWBG can be discovered at the LHC via $b g \rightarrow b A \rightarrow b Z H$ (or $b g \rightarrow b H \rightarrow b Z A$ ) process if $\left|\operatorname{Im}\left(\rho_{b b}\right)\right| \sim \mathcal{O}(0.1)$ [94]. However, the process requires that $m_{A}>m_{H}+m_{Z}$ and $\rho_{t t}$ to be negligibly small to avoid constraints from flavor physics [94]. In addition, for $m_{A}>2 m_{t}$, the process gets dilution from $A \rightarrow t \bar{t}$ decay if $\rho_{t t}$ is nonvanishing. Also, it would be extremely difficult to probe the phase of $\rho_{b b}$ at the LHC since its information is lost in $p p$ collision.

In this paper we show that the electron EDM measurement and asymmetry of CP asymmetry of the $B \rightarrow X_{s} \gamma$ decay offer exquisite probes for $\operatorname{Im}\left(\rho_{b b}\right)$. We also analyze the prospect of discovery at the LHC. In particular we study the discovery potential of $\rho_{b b}$-EWBG via $b g \rightarrow b A \rightarrow b Z h$ and $b g \rightarrow b A \rightarrow b t \bar{t}$ processes at $14 \mathrm{TeV}$ LHC. Purpose of this paper is to find possible direct and indirect signatures and correlation between them in probing the parameter space for the $\rho_{b b}$-EWBG.

\footnotetext{
${ }^{2}$ For pedagogical reviews on $2 \mathrm{HDM}$ see e.g. refs. [90, 91] and references there in.
} 
Induced by $\rho_{b b}$, the $b g \rightarrow b A \rightarrow b Z h$ process can be searched at the LHC via $p p \rightarrow$ $b A+X \rightarrow b Z h+X\left(X\right.$ is inclusive activities) followed by $Z \rightarrow \ell^{+} \ell^{-}(\ell=e, \mu)$ and $h \rightarrow b \bar{b}$ decays, constituting same flavor opposite sign dilepton pair and three $b$-tagged jets. While the $b g \rightarrow b A \rightarrow b Z h$ process can be induced $\rho_{b b}$, the $b g \rightarrow b A \rightarrow b t \bar{t}$ process requires both $\rho_{b b}$ and $\rho_{t t}$ to be nonvanishing. The latter process can be searched via $p p \rightarrow b A+X \rightarrow b t \bar{t}+X$ with at least one top decays semileptonically, constituting three $b$-tagged jets, at least one charged lepton $(e$ and $\mu)$ and missing transverse energy signature (denoted as $3 b 1 \ell$ process). These processes provide the sensitive probes for the parameter space of $\rho_{b b}$-EWBG, which is complementary to ref. [94].

For the sake of completeness we also investigate the discovery prospect of the $g g \rightarrow$ $t \bar{t} A \rightarrow t \bar{t} b \bar{b}$ process, which is induced by nonzero $\rho_{b b}$ and $\rho_{t t}$. At the LHC the process can be searched via $p p \rightarrow t \bar{t} A+X \rightarrow t \bar{t} b \bar{b}+X$, with at least one top decaying semileptonically. As $\rho_{t t}$ gets involved in both $b g \rightarrow b A \rightarrow b t \bar{t}$ and $g g \rightarrow t \bar{t} A \rightarrow t \bar{t} b \bar{b}$, the processes would provide complementary probes also for $\rho_{t t}$-EWBG.

In the following, we outline the formalism in section 2 , followed by a detailed discussion on the available parameter space and potential indirect probes in section 3 . We discuss the discovery potential of $\rho_{b b}$-EWBG at the LHC in section 4 and summarize our results with some outlook in section 5 .

\section{Framework}

The particle content of g2HDM is the SM plus additional Higgs doublet. In general, this model induces flavor-changing neutral current (FCNC) processes mediated by the neutral Higgs bosons at tree level. It is common to impose a $Z_{2}$ symmetry to suppress the FCNC processes to be consistent with various flavor physics data. Though this setup works well, having the $Z_{2}$ symmetry implies that the model has some specific UV theories such as supersymmetric models. Since we do not try to connect the model to any specific UV completions, we do not impose the $Z_{2}$ symmetry or something similar, which enables us to discuss physics at $\mathcal{O}(100) \mathrm{GeV}$ scale in wider perspective. In this bottom-up approach, the tree-level FCNC processes are possible as long as the experimental data allow, and sources of $\mathrm{CP}$ violation are much richer than $2 \mathrm{HDMs}$ with some discrete symmetries.

The most general two Higgs doublet potential can be written in the Higgs basis as $[95,96]$

$$
\begin{aligned}
V\left(\Phi, \Phi^{\prime}\right)= & \mu_{11}^{2}|\Phi|^{2}+\mu_{22}^{2}\left|\Phi^{\prime}\right|^{2}-\left(\mu_{12}^{2} \Phi^{\dagger} \Phi^{\prime}+\text { H.c. }\right)+\frac{\eta_{1}}{2}|\Phi|^{4}+\frac{\eta_{2}}{2}\left|\Phi^{\prime}\right|^{4}+\eta_{3}|\Phi|^{2}\left|\Phi^{\prime}\right|^{2} \\
& +\eta_{4}\left|\Phi^{\dagger} \Phi^{\prime}\right|^{2}+\left[\frac{\eta_{5}}{2}\left(\Phi^{\dagger} \Phi^{\prime}\right)^{2}+\left(\eta_{6}|\Phi|^{2}+\eta_{7}\left|\Phi^{\prime}\right|^{2}\right) \Phi^{\dagger} \Phi^{\prime}+\text { H.c. }\right]
\end{aligned}
$$

Each Higgs doublet fields is expressed as

$$
\Phi=\left(\begin{array}{c}
G^{+} \\
\frac{1}{\sqrt{2}}\left(v+h+i G^{0}\right)
\end{array}\right), \quad \Phi^{\prime}=\left(\begin{array}{c}
H^{+} \\
\frac{1}{\sqrt{2}}(A+H)
\end{array}\right)
$$


where $v(\simeq 246 \mathrm{GeV})$ is the vacuum expectation value, $h$ is the SM-like Higgs boson, $G^{0, \pm}$ are the Nambu-Goldstone bosons, $H$ and $A$ are the CP-even and -odd Higgs bosons, respectively, and $H^{ \pm}$are the charged Higgs bosons. From the minimization condition with respect to $\Phi$, it follows that $\mu_{11}^{2}=-\eta_{1} v^{2} / 2$. For simplicity, we assume CP-conserving Higgs sector at tree level. ${ }^{3}$ The second minimization condition with respect to $\Phi^{\prime}$ gives $\mu_{12}^{2}=\eta_{6} v^{2} / 2$.

The mixing angle $\gamma$ between the CP-even bosons $h$ and $H$ satisfies the relations [96]

$$
\cos ^{2} \gamma=\frac{\eta_{1} v^{2}-m_{h}^{2}}{m_{H}^{2}-m_{h}^{2}}, \quad \sin 2 \gamma=\frac{2 \eta_{6} v^{2}}{m_{H}^{2}-m_{h}^{2}} .
$$

An alignment limit is defined as $c_{\gamma}=0$ and $s_{\gamma}=-1$, where $c_{\gamma}$ and $s_{\gamma}$ are shorthands for $\cos \gamma$ and $\sin \gamma$ respectively. One can express the masses of $h, H, A$ and $H^{ \pm}$in terms of the parameters in eq. (2.1):

$$
\begin{aligned}
m_{h, H}^{2} & =\frac{1}{2}\left[m_{A}^{2}+\left(\eta_{1}+\eta_{5}\right) v^{2} \mp \sqrt{\left[m_{A}^{2}+\left(\eta_{5}-\eta_{1}\right) v^{2}\right]^{2}+4 \eta_{6}^{2} v^{4}}\right], \\
m_{A}^{2} & =\frac{1}{2}\left(\eta_{3}+\eta_{4}-\eta_{5}\right) v^{2}+\mu_{22}^{2}, \\
m_{H^{ \pm}}^{2} & =\frac{1}{2} \eta_{3} v^{2}+\mu_{22}^{2} .
\end{aligned}
$$

Note that in the alignment limit, one has $m_{h}^{2}=\eta_{1} v^{2}$ and $m_{H}^{2}=m_{A}^{2}+\eta_{5} v^{2}=\left(\eta_{3}+\eta_{4}+\right.$ $\left.\eta_{5}\right) v^{2} / 2+\mu_{22}^{2}$. In contrast to $m_{h}$, the masses of the extra Higgs bosons are controlled by $\eta_{i} v^{2}$ and $\mu_{22}^{2}$, where $\eta_{i}$ denotes some linear combinations of the $\eta$ couplings. As is well known, magnitudes of the heavy Higgs loop contributions can become sizable if $\eta_{i} v^{2} \gtrsim \mu_{22}^{2}$, which is necessary for achieving the strong first-order EWPT.

The CP-even scalars $h, H$ and CP-odd scalar $A$ couple to fermions by [95]

$$
\begin{aligned}
\mathcal{L}= & -\frac{1}{\sqrt{2}} \sum_{F=U, D, L} \bar{F}_{i}\left[\left(-\lambda_{i j}^{F} s_{\gamma}+\rho_{i j}^{F} c_{\gamma}\right) h+\left(\lambda_{i j}^{F} c_{\gamma}+\rho_{i j}^{F} s_{\gamma}\right) H-i \operatorname{sgn}\left(Q_{F}\right) \rho_{i j}^{F} A\right] P_{R} F_{j} \\
& -\bar{U}_{i}\left[\left(V \rho^{D}\right)_{i j} P_{R}-\left(\rho^{U \dagger} V\right)_{i j} P_{L}\right] D_{j} H^{-} \bar{\nu}_{i} \rho_{i j}^{L} P_{R} L_{j} H^{+}+\text {H.c. }
\end{aligned}
$$

where $P_{L, R} \equiv\left(1 \mp \gamma_{5}\right) / 2, i, j=1,2,3$ are generation indices, $V$ is CKM matrix, and $U=(u, c, t), D=(d, s, b), L=(e, \mu, \tau)$ and $\nu=\left(\nu_{e}, \nu_{\mu}, \nu_{\tau}\right)$ are in vectors in flavor space. The matrices $\lambda_{i j}^{F}\left(=\sqrt{2} m_{i}^{F} / v\right)$ are real and diagonal, whereas $\rho_{i j}^{F}$ are in general complex and non-diagonal.

Purpose of this paper is to probe the parameter space for EWBG driven by the extra bottom Yukawa $\rho_{b b}$. It is found that a successful EWBG requires $\left|\operatorname{Im}\left(\rho_{b b}\right)\right| \gtrsim 0.058$ [85]. In the following we shall show that the parameter space receives meaningful constraints from several direct and indirect searches. The most stringent constraint on $\operatorname{Im}\left(\rho_{b b}\right)$ arises from electron EDM and $\Delta \mathcal{A}_{\mathrm{CP}}$ of $\mathcal{B}\left(B \rightarrow X_{s} \gamma\right)$. In addition, coupling strength measurements of $h$ and heavy Higgs searches at the LHC would also provide the complementary probes. In

\footnotetext{
${ }^{3}$ Since we have CP violation in the Yukawa sector as delineated below, its effect appears in the Higgs spectrum at one-loop level and CP-even and -odd Higgs boson mix with each other. Nevertheless, such a one-loop induced mixing is so small that $\{h, H, A\}$ can be regarded as the mass eigenstates to a good approximation.
} 
addition to these constraints, we also study potential signatures at the LHC. We primarily focus on three searches at the $14 \mathrm{TeV}$ LHC $b g \rightarrow b A \rightarrow b Z h,{ }^{4} g g \rightarrow t \bar{t} A \rightarrow t \bar{t} b \bar{b}$ and $b g \rightarrow$ $b A \rightarrow b t \bar{t}$ (for the discussion on latter two processes see also refs. $[105]^{5}$ ). Induced by $\rho_{b b}$ the $b g \rightarrow b A \rightarrow b Z h$ process can be searched at the LHC if $c_{\gamma}$ is nonzero and $m_{A}>m_{h}+m_{Z}$. On the other hand, $g g \rightarrow t \bar{t} A \rightarrow t \bar{t} b \bar{b}$ process requires $\rho_{t t}$ and $\rho_{b b}$ both nonvanishing with mild dependence on $c_{\gamma}$. The final process $b g \rightarrow b A \rightarrow b t \bar{t}$ also depends both on $\rho_{b b}$ and $\rho_{t t}$ but needs $m_{A}>2 m_{t}$. Together with electron EDM and $\Delta \mathcal{A}_{\mathrm{CP}}$ of $\mathcal{B}\left(B \rightarrow X_{s} \gamma\right)$, these processes can probe significant part of the parameter space for $\rho_{b b}$-EWBG.

Note that complex $\rho_{t t}$ can provide a more robust mechanism for EWBG $[84,86]$. One may also have complementary probes for the $\rho_{t t}$-EWBG from $g g \rightarrow t \bar{t} A \rightarrow t \bar{t} b \bar{b}$ and $b g \rightarrow b A \rightarrow b t \bar{t}$ processes. Nonvanishing $\rho_{t t}$ motivates the conventional $g g \rightarrow H \rightarrow$ $t \bar{t}[106,107]^{6}$ search or $g g \rightarrow t \bar{t} A / H \rightarrow t \bar{t} t \bar{t}[101-105],{ }^{7}$ i.e., the four top search. Though the former process suffers from large interference with the overwhelming QCD $g g \rightarrow t \bar{t}$ background [108], recent searches performed by both ATLAS [106] and CMS [107] found some sensitivity. When both $\rho_{b b}$ and $\rho_{t t}$ are nonzero, one may also have $g g \rightarrow b \bar{b} A / H \rightarrow$ $b \bar{b} t \bar{t}$, which are covered in refs. [101-105].

\section{Parameter space}

Let us find the allowed parameter space for $m_{A}, m_{H}$ and $m_{H^{ \pm}}$. The parameters in eq. (2.1) are required to satisfy perturbativity, tree-level unitarity and vacuum stability conditions, for which we utilized the public tool $2 \mathrm{HDMC}$ [112]. We express the quartic couplings $\eta_{1}$, $\eta_{3-6}$ in terms of $m_{h}, m_{H}, m_{H^{ \pm}}, m_{A}, \mu_{22}, \gamma$, and $v$ as [95]

$$
\begin{aligned}
\eta_{1} & =\frac{m_{h}^{2} s_{\gamma}^{2}+m_{H}^{2} c_{\gamma}^{2}}{v^{2}}, \\
\eta_{3} & =\frac{2\left(m_{H^{ \pm}}^{2}-\mu_{22}^{2}\right)}{v^{2}}, \\
\eta_{4} & =\frac{m_{h}^{2} c_{\gamma}^{2}+m_{H}^{2} s_{\gamma}^{2}-2 m_{H^{ \pm}}^{2}+m_{A}^{2}}{v^{2}}, \\
\eta_{5} & =\frac{m_{H}^{2} s_{\gamma}^{2}+m_{h}^{2} c_{\gamma}^{2}-m_{A}^{2}}{v^{2}}, \\
\eta_{6} & =\frac{\left(m_{h}^{2}-m_{H}^{2}\right)\left(-s_{\gamma}\right) c_{\gamma}}{v^{2}} .
\end{aligned}
$$

The quartic couplings $\eta_{2}$ and $\eta_{7}$ do not enter scalar masses, nor the mixing angle $\gamma$. Therefore in our analysis we take $v, m_{h}$, and $\gamma, m_{A}, m_{H}, m_{H^{ \pm}}, \mu_{22}, \eta_{2}, \eta_{7}$ as the phenomenological parameters. Further, to save computation time, we randomly generated these parameters in the following ranges: $\mu_{22} \in[0,1000] \mathrm{GeV}, m_{A} \in[250,600] \mathrm{GeV}$, $m_{H} \in[250,600] \mathrm{GeV}, m_{H^{ \pm}} \in[250,600] \mathrm{GeV}, \eta_{2} \in[0,6], \eta_{7} \in[-6,6]$, while satisfying

\footnotetext{
${ }^{4}$ Discussions on similar processes can also be found in refs. [97-100].

${ }^{5}$ For recent discussions see e.g. refs. [101-104].

${ }^{6}$ For a recent reference see also ref. [108].

${ }^{7}$ See e.g. for a non exhaustive list refs. [109-111].
} 

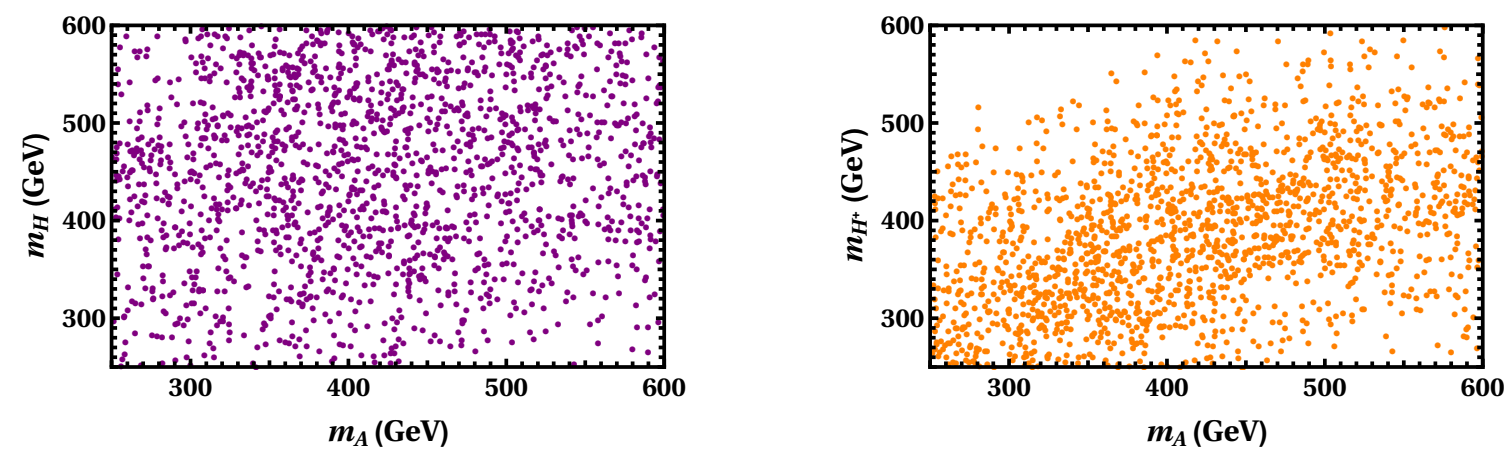

Figure 1. Scanned points plotted in $m_{A^{-}} m_{H}$ (left) and $m_{A^{-}} m_{H^{ \pm}}$(right) plane. Here the scanned points satisfy the tree level unitarity, perturbativity and positivity conditions as well as the $T$ parameter constraint. However, not all points lead to the strong first-order EWPT.

$m_{h}=125 \mathrm{GeV}$ with $c_{\gamma}=0.1 .^{8}$ The randomly generated parameters are then fed into 2HDMC for scanning. 2HDMC utilizes [112] $m_{H^{ \pm}}$and $\Lambda_{1-7}$ as the input parameters in the Higgs basis whereas $v \simeq 246 \mathrm{GeV}$. In order to match the $2 \mathrm{HDMC}$ convention, we identify $\eta_{1-7}$ as $\Lambda_{1-7}$ and, take $-\pi / 2 \leq \gamma \leq \pi / 2$. For the positivity conditions of the Higgs potential of eq. (2.1), the parameter $\eta_{2}>0$ along with other more involved conditions implemented in 2HDMC. We further conservatively demand $\left|\eta_{i}\right| \leq 6$.

Next we impose the stringent oblique $T$ parameter [113] constraint, which restricts hierarchical structures among the scalar masses $m_{H}, m_{A}$ and $m_{H^{ \pm}}[114,115]$, and hence $\eta_{i}$ s. Utilizing the expression given in ref. [115] the points that passed unitarity, perturbativity and positivity conditions from $2 \mathrm{HDMC}$, are further required to satisfy the $T$ parameter constraint within the $2 \sigma$ error [116]..$^{9}$ These points are denoted as "scanned points". We plot the scanned points in the $m_{A}{ }^{-} m_{H}$ and $m_{A^{-}} m_{H^{ \pm}}$planes in the left and right panels of figure 1, which illustrates that significant amounts of the allowed points exists. A more detailed discussions on the scanning procedure can be found in refs. [118, 119]. At this point, we have not yet required that EWPT should be strongly first order, and not all points are compatible with the $\rho_{b b}$ EWBG.

To find the constraints on $\rho_{b b}$ and $\rho_{t t}$ and, subsequently analyze the potential of future probes we choose three benchmark points (BPs) from the scanned points in figure 1, which are summarized in table 1 . Here, we also demand that the chosen parameter sets give rise to the strong first-order EWPT. The $\mathrm{BP} a$ and $\mathrm{BP} b$ are chosen such that $m_{A}<2 m_{t}$. Since there is no suppression from $\mathcal{B}(A \rightarrow t \bar{t})$, such a choice would enhance the discovery potential of $b g \rightarrow b A \rightarrow b Z h$ and $g g \rightarrow t \bar{t} A \rightarrow t \bar{t} b \bar{b}$. For the BP $c$, where $m_{A}>2 m_{t}$, the $b g \rightarrow b A \rightarrow b t \bar{t}$ process ${ }^{10}$ can provide additional probes for the parameter space. Further, for all three BPs, $A$ is assumed to be lighter than $H$ and $H^{ \pm}$to forbid $A \rightarrow Z H$ and

\footnotetext{
${ }^{8}$ Note that, for successful $\rho_{b b}$ induced EWBG, one requires non vanishing $c_{\gamma}$ as discussed in ref. [85]. It was shown that for $c_{\gamma} \sim 0.1$, current data still allows $\operatorname{Im}\left(\rho_{b b}\right) \sim 0.15-0.2$, while $\left|\operatorname{Im}\left(\rho_{b b}\right)\right| \gtrsim 0.058$ is sufficient to account for the observed BAU [85].

${ }^{9}$ The latest value of $T$ parameter is obtained from ref. [117].

${ }^{10}$ Note that for $\mathrm{BP} a$ and $\mathrm{BP} b$ one may have $b g \rightarrow b H \rightarrow b \bar{b} t \bar{t}$, which can resemble similar final state topologies as in $b g \rightarrow b A \rightarrow b t \bar{t}$. This would be discussed in the section 4.2 .
} 


\begin{tabular}{|c|c|c|c|c|c|c|c|c|c|c|c|}
\hline BP & $\eta_{1}$ & $\eta_{2}$ & $\eta_{3}$ & $\eta_{4}$ & $\eta_{5}$ & $\eta_{6}$ & $\eta_{7}$ & $\begin{array}{c}m_{H^{ \pm}} \\
(\mathrm{GeV})\end{array}$ & $\begin{array}{c}m_{A} \\
(\mathrm{GeV})\end{array}$ & $\begin{array}{c}m_{H} \\
(\mathrm{GeV})\end{array}$ & $\frac{\mu_{22}^{2}}{v^{2}}$ \\
\hline & & & & & & & & & & & \\
$a$ & 0.282 & 2.034 & 4.053 & -1.039 & 1.343 & -0.243 & 1.231 & 391 & 285 & 405 & 0.5 \\
$b$ & 0.289 & 1.959 & 4.064 & -0.418 & 1.56 & -0.316 & -1.216 & 414 & 334 & 456 & 0.8 \\
$c$ & 0.303 & 0.413 & 5.129 & -0.477 & 1.534 & -0.455 & 0.457 & 508 & 444 & 541 & 1.7 \\
\hline
\end{tabular}

Table 1. Parameter values of three benchmark points chosen from the scanned points in figure 1, which are consistent with the strong first-order EWPT.
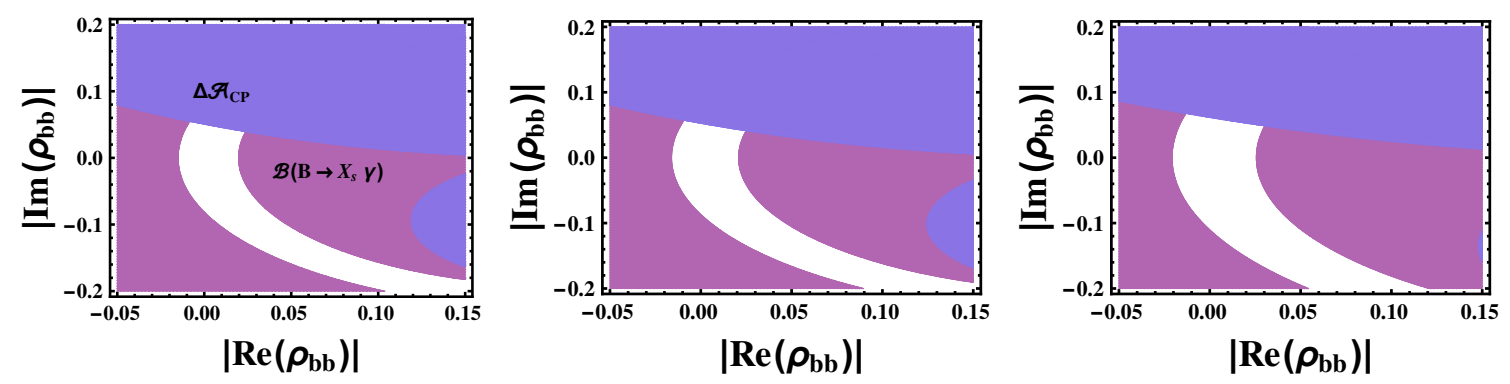

Figure 2. The constraints on $\rho_{b b}$ from $\mathcal{B}\left(B \rightarrow X_{s} \gamma\right)$ (purple) and $\Delta \mathcal{A}_{\mathrm{CP}}$ (blue) measurements for the $\mathrm{BP} a$ (left), $\mathrm{BP} b$ (middle) and $\mathrm{BP} b$ (right) respectively. All three figures are generated assuming $\rho_{t t}=0.5$. See text for details.

$A \rightarrow H^{ \pm} W^{\mp}$ decays and boost the discovery potential of these processes to some extent. Heavier $A$ are indeed possible, but the cross sections are reduced due to rapid fall in the parton luminosity. We also remark that one requires [4-14] sub-TeV $m_{A}, m_{H^{ \pm}}$and $m_{H}$ for the strong first-order EWPT, which is required for conventional sub-TeV EWBG [15-77] (for high-scale EWBG, see, e.g., refs. [87-89]).

In the following we will scrutinize the relevant constraints on $\rho_{b b}$ and $\rho_{t t}$. For simplicity, we assume that $\rho_{i j}$ except for $\rho_{b b}, \rho_{t t}$ and $\rho_{e e}$ are negligibly small so as not to affect our main discussion. The impacts of nonzero $\rho_{i j}$ would be discussed later part of the paper.

\subsection{Flavor constraints}

There exist several constraints from flavor physics that restricts the parameter space. In particular, the following three observables are relevant: (i) the branching ratio measurement of $B \rightarrow X_{s} \gamma\left(\mathcal{B}\left(B \rightarrow X_{s} \gamma\right)\right)$, (ii) the asymmetry of the CP asymmetry between the charged and neutral $B \rightarrow X_{s} \gamma$ decays $\left(\Delta \mathcal{A}_{\mathrm{CP}}\right)$ and (iii) the $B_{q}-\bar{B}_{q}(q=d, s)$ mixings.

Let us first focus on $\mathcal{B}\left(B \rightarrow X_{s} \gamma\right)$. Non-zero $\rho_{b b}$ and $\rho_{t t}$ modify $\mathcal{B}\left(B \rightarrow X_{s} \gamma\right)$ via top quark and charged Higgs boson loop. The modification is parametrized by the (LO) Wilson coefficients $C_{7,8}^{(0)}$ at the matching scale $\mu=m_{W}$

$$
C_{7,8}^{(0)}\left(m_{W}\right)=F_{7,8}^{(1)}\left(x_{t}\right)+\delta C_{7,8}^{(0)}\left(\mu_{W}\right)
$$

where, $\bar{m}_{t}\left(m_{W}\right)$ is the top quark $\overline{\mathrm{MS}}$ running mass at the $m_{W}$ scale with $x_{t}=$ $\left(\bar{m}_{t}\left(m_{W}\right) / m_{W}\right)^{2}$. The expression for $F_{7,8}^{(1)}(x)$ can be found in the refs. [120, 121], whereas 
$\delta C_{7,8}^{(0)}\left(\mu_{W}\right)$ the LO (leading order) charged Higgs contributions. At LO, $\delta C_{7,8}^{(0)}\left(\mu_{W}\right)$ is expressed as [122]

$$
\delta C_{7,8}^{(0)}\left(m_{W}\right) \simeq \frac{\left|\rho_{t t}\right|^{2}}{3 \lambda_{t}^{2}} F_{7,8}^{(1)}\left(y_{H^{+}}\right)-\frac{\rho_{t t} \rho_{b b}}{\lambda_{t} \lambda_{b}} F_{7,8}^{(2)}\left(y_{H^{+}}\right),
$$

with $y_{H^{+}}=\left(\bar{m}_{t}\left(m_{W}\right) / m_{H^{+}}\right)^{2}$ while, the full expression for $F_{7,8}^{(2)}\left(y_{H^{+}}\right)$can be found in ref. [120]. The current world average of $\mathcal{B}\left(B \rightarrow X_{s} \gamma\right)_{\text {exp }}$ extrapolated to the photon-energy cut $E_{0}=1.6 \mathrm{GeV}$ is found by the HFLAV Collaboration to be $(3.32 \pm 0.15) \times 10^{-4}[123]$. The next-to-next-to LO (NNLO) $\mathcal{B}\left(B \rightarrow X_{s} \gamma\right)$ prediction in the SM for the same photonenergy cut is $(3.36 \pm 0.23) \times 10^{-4}$ [124]. In order to find the constraint, we adopt the prescription outlined in ref. [125] and define

$$
R_{\exp }=\frac{\mathcal{B}\left(B \rightarrow X_{s} \gamma\right)_{\exp }}{\mathcal{B}\left(B \rightarrow X_{s} \gamma\right)_{\mathrm{SM}}}
$$

Based on our LO Wilson coefficients, we further express

$$
R_{\text {theory }}=\frac{\mathcal{B}\left(B \rightarrow X_{s} \gamma\right)_{\mathrm{g} 2 \mathrm{HDM}}}{\mathcal{B}\left(B \rightarrow X_{s} \gamma\right)_{\mathrm{SM}}},
$$

and take $m_{W}$ and $\bar{m}_{b}\left(m_{b}\right)$ respectively as the matching scale and the low-energy scales. Finally, we demand $R_{\text {theory }}$ to remain within the $2 \sigma$ error of $R_{\text {exp }}$. In figure 2 the excluded regions are shown as the purple shaded regions in the $\operatorname{Re}\left(\rho_{b b}\right)-\operatorname{Im}\left(\rho_{b b}\right)$ plane for three BPs. Here, we assume $\rho_{t t}=0.5$. Flavor constraints on $\rho_{t t}$ is moderately strong, with $B_{d, s^{-}} \bar{B}_{d, s}$ mixings providing the most stringent constraint on $\rho_{t t}$ for $500 \lesssim m_{H^{+}} \lesssim 650 \mathrm{GeV}$, which is the ballpark mass ranges of $m_{H^{ \pm}}$for all the three BPs. The $B_{q^{-}} \bar{B}_{q}$ mixing amplitude $M_{12}^{q}$ receives modification from the charged Higgs and $W$ bosons loop with $t$ quark. Utilizing the expression for $B_{q}-\bar{B}_{q}$ mixing in type-II $2 \mathrm{HDM}$ [126], it is found in ref. [122] that

$$
\frac{M_{12}^{q}}{M_{12}^{q} \mathrm{SM}}=1+\frac{I_{W H}\left(y_{W}, y_{H}, x\right)+I_{H H}\left(y_{H}\right)}{I_{W W}\left(y_{W}\right)},
$$

where $y_{i}=m_{t}^{2} / m_{i}^{2}\left(i=W, H^{ \pm}\right)$and $x=m_{H^{ \pm}}^{2} / m_{W}^{2}$ with $m_{t}$ and $m_{W}$ being the masses of the top quark and $W$ bosons. The expressions for $I_{W W}, I_{W H}$ and $I_{H H}$ are respectively given by [122]

$$
\begin{aligned}
I_{W W}= & 1+\frac{9}{1-y_{W}}-\frac{6}{\left(1-y_{W}\right)^{2}}-\frac{6}{y_{W}}\left(\frac{y_{W}}{1-y_{W}}\right)^{3} \ln y_{W}, \\
I_{W H} \simeq & \left(\frac{\rho_{t t}^{*}}{\lambda_{t}}+\frac{V_{c b} \rho_{c t}^{*}}{V_{t b} \lambda_{t}}\right)\left(\frac{\rho_{t t}}{\lambda_{t}}+\frac{V_{c q}^{*} \rho_{c t}}{V_{t q}^{*} \lambda_{t}}\right) y_{H} \\
& \times\left[\frac{(2 x-8) \ln y_{H}}{(1-x)\left(1-y_{H}\right)^{2}}+\frac{6 x \ln y_{W}}{(1-x)\left(1-y_{W}\right)^{2}}-\frac{8-2 y_{W}}{\left(1-y_{W}\right)\left(1-y_{H}\right)}\right], \\
I_{H H} \simeq & \left(\frac{\rho_{t t}^{*}}{\lambda_{t}}+\frac{V_{c b} \rho_{c t}^{*}}{V_{t b} \lambda_{t}}\right)^{2}\left(\frac{\rho_{t t}}{\lambda_{t}}+\frac{V_{c q}^{*} \rho_{c t}}{V_{t q}^{*} \lambda_{t}}\right)^{2}\left(\frac{1+y_{H}}{\left(1-y_{H}\right)^{2}}+\frac{2 y_{H} \ln y_{H}}{\left(1-y_{H}\right)^{3}}\right) y_{H} .
\end{aligned}
$$

For $\left|\rho_{t t}\right| \sim \mathcal{O}(1)$ coupling $\rho_{c t}$ is strongly constrained due to $\left|V_{c q} / V_{t q}\right| \sim 25(q=d, s)$ enhancement [122], as can be seen from eqs. (3.12) and (3.13). As we are primarily interested in the parameter space where $\rho_{t t}$ is $\mathcal{O}(1)$, we turn off $\rho_{c t}$ throughout our paper for 
simplicity. The 2018 summer results of UTfit finds [127]: ${ }^{11}$

$$
\begin{aligned}
& C_{B_{d}} \in 1.05 \pm 0.11, \\
& C_{B_{s}} \in 1.110 \pm 0.090, \\
& \phi_{B_{d}} \in-2.0 \pm 1.8 \quad\left[\mathrm{in}^{\circ}\right], \\
& \phi_{B_{s}} \in 0.42 \pm 0.89 \quad\left[\mathrm{in}^{\circ}\right] .
\end{aligned}
$$

with $M_{12}^{q} / M^{q \mathrm{SM}}=C_{B_{q}} e^{2 i \phi_{B_{q}}}$. Under the assumption on the $\rho_{i j}^{F}$ couplings made in our analysis, we have $M_{12}^{q} / M^{q} \mathrm{SM}=C_{B_{q}}$. Allowing $2 \sigma$ errors on $C_{B_{d}}$ and $C_{B_{s}}$ we find that $B_{s, d}-\bar{B}_{d, s}$ mixings exclude $\left|\rho_{t t}\right| \gtrsim 0.9$ for $\mathrm{BP} a$ and $\mathrm{BP} b$ and, $\left|\rho_{t t}\right| \gtrsim 1$ for $\mathrm{BP} c$.

One of the most powerful probes of $\operatorname{Im}\left(\rho_{b b}\right)$ is the direct CP asymmetry $\mathcal{A}_{\mathrm{CP}}$ [129] of $B \rightarrow X_{s} \gamma$. It is advocated in ref. [130], however, that $\Delta \mathcal{A}_{\mathrm{CP}}$ is even more sensitive to the CP-violating couplings, which is defined as [130]

$$
\Delta \mathcal{A}_{\mathrm{CP}}=\mathcal{A}_{B^{-} \rightarrow X_{s}^{-} \gamma}-\mathcal{A}_{B^{0} \rightarrow X_{s}^{0} \gamma} \approx 4 \pi^{2} \alpha_{s} \frac{\tilde{\Lambda}_{78}}{m_{b}} \operatorname{Im}\left(\frac{C_{8}}{C_{7}}\right),
$$

where $\tilde{\Lambda}_{78}$ and $\alpha_{s}$ denote a hadronic parameter and the strong coupling constant at $\bar{m}_{b}\left(m_{b}\right)$ scale, respectively. One expects that $\tilde{\Lambda}_{78}$ has a similar scale of $\Lambda_{\mathrm{QCD}}$. In ref. [130], it is found that $17 \mathrm{MeV} \leq \tilde{\Lambda}_{78} \leq 190 \mathrm{MeV}$. On the other hand, recently Belle measured $\Delta \mathcal{A}_{\mathrm{CP}}=(+3.69 \pm 2.65 \pm 0.76) \%[131]$, where the first uncertainty is statistical while the second one is systematic. Allowing $2 \sigma$ error on the Belle measurement, we show the regions excluded by $\Delta \mathcal{A}_{\mathrm{CP}}$ in blue shade in figures 2 for the three BPs. Here, we choose the average value of $\tilde{\Lambda}_{78}$ i.e., $89 \mathrm{MeV}$ for illustration. We stress that the constraint shown in figures 2 depends heavily on the value of $\tilde{\Lambda}_{78}$. The larger $\tilde{\Lambda}_{78}$ would make the constraint stronger. We also remark that we utilize the LO Wilson coefficients in eq. (3.6) as a first approximation for simplicity. Note that the excluded regions by $\Delta \mathcal{A}_{\mathrm{CP}}$ measurement in figure 2 is asymmetric and constrains positive $\operatorname{Im}\left(\rho_{b b}\right)$ more stringently. This is solely due to our choice of $\rho_{t t}=0.5$. If we take $\rho_{t t}=-0.5$, the blue shaded regions would flip and exclude the negative regions of $\operatorname{Im}\left(\rho_{b b}\right)$.

We note in passing that if $\rho_{t t}$ is also complex, $\Delta \mathcal{A}_{\mathrm{CP}}$ can be zero if the complex phases of $\rho_{t t}$ and $\rho_{b b}$ are aligned, i.e., $\operatorname{Im}\left(\rho_{t t} \rho_{b b}\right)=0$, equivalently, $\operatorname{Re} \rho_{b b} / \operatorname{Re} \rho_{t t}=-\operatorname{Im} \rho_{b b} / \operatorname{Im} \rho_{t t}$. Such a phase alignment is discussed in ref. [86].

\subsection{EDMs}

The complex phase of $\rho_{b b}$ is severely constrained by EDMs of the electron, neutron, and atoms, etc. Currently, the most stringent experimental bound comes from EDM of thorium monoxide ( $\mathrm{ThO})$, which is approximately given by

$$
d_{\mathrm{ThO}}=d_{e}+\alpha_{\mathrm{ThO}} C_{S},
$$

where $d_{e}$ is the electron EDM and $C_{S}$ is the coefficient of the nuclear spin-independent interaction (NSID), which are respectively defined as

$$
\mathcal{L}_{\mathrm{EDM}}=-\frac{i}{2} d_{e} F^{\mu \nu} \bar{e} \sigma_{\mu \nu} \gamma_{5} e, \quad \mathcal{L}_{e N}^{\mathrm{NSID}}=-\frac{G_{F}}{\sqrt{2}} C_{S}(\bar{N} N)\left(\bar{e} i \gamma_{5} e\right),
$$

\footnotetext{
${ }^{11}$ The New Physics Fit results of 2018 Summer can be found at ref. [128].
} 


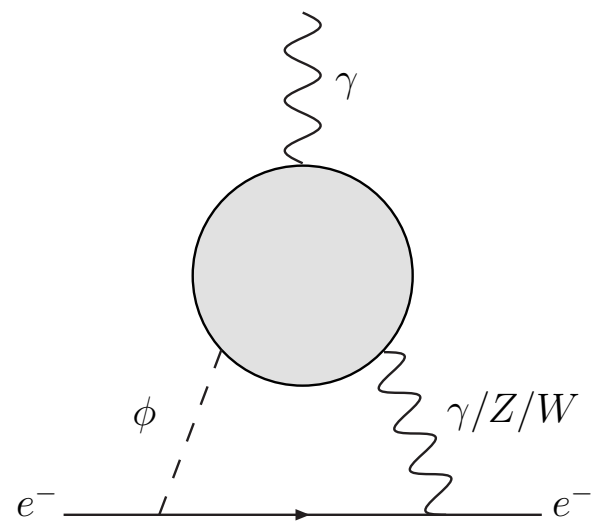

Figure 3. Two-loop Barr-Zee diagrams contributing to the electron EDM, where $\phi=h, H, A, H^{ \pm}$. The shaded loop collectively represents the scalar, fermion and gauge boson loops. The total contribution is given by their sum, $d_{e}=d_{e}^{\phi \gamma}+d_{e}^{\phi Z}+d_{e}^{\phi W}$.

where $F^{\mu \nu}$ denotes the field strength tensor of electromagnetism and $G_{F}$ is the Fermi coupling constant. The coefficient $\alpha_{\mathrm{ThO}}$ is estimated as $\alpha_{\mathrm{ThO}}=1.5 \times 10^{-20}$ [132]. The latest experimental value of $d_{\mathrm{ThO}}$ is placed by ACME Collaboration in 2018 (ACME18) as

$$
d_{\mathrm{ThO}}=(4.3 \pm 4.0) \times 10^{-30} \text { e cm, }
$$

from which under the assumption of $C_{S}=0$ the electron EDM has an upper bound of

$$
\left|d_{e}\right|<1.1 \times 10^{-29} e \mathrm{~cm} \text {. }
$$

In our scenario, $d_{e}$ is predominantly induced by two-loop Barr-Zee diagrams as depicted in figure 3 , which are decomposed into the three parts:

$$
d_{e}=d_{e}^{\phi \gamma}+d_{e}^{\phi Z}+d_{e}^{\phi W}
$$

where $\phi=h, H, A$ for the first two terms and $\phi=H^{ \pm}$for the last term. Let us denote the contribution of $i$-species to $d_{e}^{\phi \gamma}$ as $\left(d_{e}^{\phi \gamma}\right)_{i}$. If $\rho_{b b}$ is the only source of $C P$ violation, $d_{e} \simeq\left(d_{e}^{\phi \gamma}\right)_{b}$. With $\operatorname{Im} \rho_{b b}$ required by $\rho_{b b}$-EWBG mechanism, $d_{e}$ is so large that one cannot avoid the ACME18 bound as noted in ref. [85]. This fact suggests two options: (i) the alignment limit $\left(c_{\gamma} \rightarrow 0\right)$ and (ii) cancellation mechanism. As discussed in ref. [86], however, the first option may not be consistent with EWBG in g2HDM since the BAU would be suppressed with decreasing $c_{\gamma}$. We thus consider the second option. Even though we identify the parameter space for the cancellation in ref. [85], we do not show its detail there, and moreover, $d_{e}^{\phi W}$, which can come into play in the cancellation region, is missing. We therefore update our previous analysis taking all the relevant contributions into consideration.

If there exist more than two $C P$-violating phases, we could tune the parameters in such a way that $d_{e}$ becomes small. While it is nothing more than the parameter turning, we still classify the cancellation parameter space into two kind. We call a cancellation structured cancellation if it happens when the hierarchical structures of the $\rho_{i j}$ matrices 
closely resemble those of the SM Yukawa matrices, and anything else is unstructured cancellation. It is revealed in ref. [86] that the parameter space of $\rho_{t t}$-EWBG accommodates the structured cancellation. We here scrutinize the type of the cancellation in $\rho_{b b}$-EWBG.

Following a method adopted in ref. [86], we split $\left(d_{e}^{\phi \gamma}\right)_{b}$ into two parts as ${ }^{12}$

$$
\left(d_{e}^{\phi \gamma}\right)_{f}=\left(d_{e}^{\phi \gamma}\right)_{f}^{\operatorname{mix}}+\left(d_{e}^{\phi \gamma}\right)_{f}^{\text {extr }}
$$

where

$$
\begin{aligned}
\frac{\left(d_{e}^{\phi \gamma}\right)_{f}^{\operatorname{mix}}}{e}=-\frac{3 \alpha_{\mathrm{em}} Q_{f}^{2} s_{2 \gamma}}{16 \sqrt{2} \pi^{3} v}\left[\operatorname{Im}\left(\rho_{e e}\right) \Delta f_{f}+\frac{\lambda_{e}}{\lambda_{f}} \operatorname{Im}\left(\rho_{f f}\right) \Delta g_{f}\right] & \\
\frac{\left(d_{e}^{\phi \gamma}\right)_{f}^{\operatorname{extr}}}{e}=\frac{3 \alpha_{\mathrm{em}} Q_{f}^{2}}{16 \pi^{3} m_{f}} & {\left[\operatorname{Im}\left(\rho_{e e}\right) \operatorname{Re}\left(\rho_{f f}\right)\left\{c_{\gamma}^{2} f\left(\tau_{f h}\right)+s_{\gamma}^{2} f\left(\tau_{f H}\right) \pm g\left(\tau_{f A}\right)\right\}\right.} \\
& \left.\quad \operatorname{Im}\left(\rho_{f f}\right) \operatorname{Re}\left(\rho_{e e}\right)\left\{c_{\gamma}^{2} g\left(\tau_{f h}\right)+s_{\gamma}^{2} g\left(\tau_{f H}\right) \pm f\left(\tau_{f A}\right)\right\}\right]
\end{aligned}
$$

with $\alpha_{\mathrm{em}}$ and $Q_{f}$ representing the fine structure constant and electric charges of $f$, respectively, and $\Delta f_{f}=f\left(\tau_{f h}\right)-f\left(\tau_{f H}\right)$ and $\Delta g_{f}=g\left(\tau_{f h}\right)-g\left(\tau_{f H}\right)$ with $\tau_{i j}=m_{i}^{2} / m_{j}^{2} . f(\tau)$ and $g(\tau)$ are the loop functions and their explicit forms are shown in appendix A. In our notation, the sign of $e$ is positive. In the wave parenthesis in eq. (3.23), the upper sign is for up-type fermions and the lower is for down-type fermions, respectively. For $c_{\gamma} \ll 1$ and $m_{H} \simeq m_{A},\left(d_{e}^{\phi \gamma}\right)_{t, b}^{\text {extr }}$ are approximated as

$$
\begin{aligned}
\frac{\left(d_{e}^{H \gamma}\right)_{t}^{\text {extr }}}{e} & \simeq \frac{\alpha_{\mathrm{em}}}{12 \pi^{3} m_{t}} \operatorname{Im}\left(\rho_{e e} \rho_{t t}\right)\left[f\left(\tau_{t H}\right)+g\left(\tau_{t H}\right)\right] \\
\frac{\left(d_{e}^{H \gamma}\right)_{b}^{\operatorname{extr}}}{e} & \simeq \frac{\alpha_{\mathrm{em}}}{48 \pi^{3} m_{b}} \operatorname{Im}\left(\rho_{e e} \rho_{b b}^{*}\right)\left[f\left(\tau_{b H}\right)-g\left(\tau_{b H}\right)\right] .
\end{aligned}
$$

In the $\rho_{b b}$-EWBG scenario, $\operatorname{Im}\left(\rho_{e e} \rho_{t t}\right)=\rho_{t t} \operatorname{Im}\left(\rho_{e e}\right)$. To make our discussion on the cancellation mechanism simpler, we consider a case in which $\operatorname{Im}\left(\rho_{e e} \rho_{b b}^{*}\right) \simeq 0$ so that $\left(d_{e}^{\phi \gamma}\right)_{b} \simeq\left(d_{e}^{\phi \gamma}\right)_{b}^{\text {mix }}$. When $\rho_{e e}$ is nonzero, the primary contribution could be $\left(d_{e}^{\phi \gamma}\right)_{W}$ as inferred from the fact that the $\phi-\gamma-\gamma$ vertex in $d_{e}^{\phi \gamma}$ is more or less common to the $h \rightarrow 2 \gamma$ decay. Noting that the $W$-loop has only the "mix" contribution since the Higgs couplings to the $W$ bosons are the gauge couplings, one may find [133]

$$
\frac{\left(d_{e}^{H \gamma}\right)_{W}}{e}=\frac{\left(d_{e}^{H \gamma}\right)_{W}^{\operatorname{mix}}}{e}=\frac{\alpha_{\mathrm{em}} s_{2 \gamma}}{64 \sqrt{2} \pi^{3} v} \operatorname{Im}\left(\rho_{e e}\right) \Delta \mathcal{J}_{W}^{\gamma},
$$

where $\Delta \mathcal{J}_{W}^{\gamma}=\mathcal{J}_{W}^{\gamma}\left(m_{h}\right)-\mathcal{J}_{W}^{\gamma}\left(m_{H}\right)$ (for explicit form of $\mathcal{J}_{W}^{\gamma}$, see appendix A). From the condition of $\left(d_{e}^{\phi \gamma}\right)_{t}+\left(d_{e}^{\phi \gamma}\right)_{b}+\left(d_{e}^{\phi \gamma}\right)_{W}=0$, it follows that

$$
\frac{\operatorname{Im}\left(\rho_{e e}\right)}{\operatorname{Im}\left(\rho_{b b}\right)}=-\frac{s_{2 \gamma} \Delta g_{b} / 4}{s_{2 \gamma}\left[\Delta f_{t}+\Delta f_{b} / 4-(3 / 16) \Delta \mathcal{J}_{W}^{\gamma}\right]+2 \rho_{t t}\left[f\left(\tau_{t H}\right)+g\left(\tau_{t H}\right)\right] / \lambda_{t}} \equiv-c \times \frac{\lambda_{e}}{\lambda_{b}} .
$$

It is found that $c=1.0 \times 10^{-3}$ for $c_{\gamma}=0.1, \rho_{t t}=0.5, m_{h}=125 \mathrm{GeV}$ and $m_{H}=405 \mathrm{GeV}$. Therefore, the cancellation is possible but unstructured since $c$ deviates much from the unity

\footnotetext{
${ }^{12}$ By convention in this paper, the sign of $\gamma$ is opposite to that in ref. [86].
} 
as opposed to the $\rho_{t t}$ EWBG scenario [86]. Once this accidental cancellation happens, other contributions could become relevant. On the grounds of dimensional analysis, one can find that $d_{e}^{\phi Z}$ is suppressed by the $Z$ boson coupling to the electron, $g_{Z e e}=1 / 4-\sin \theta_{W} \simeq$ 0.02 with $\theta_{W}$ representing the weak mixing angle, while $d_{e}^{\phi W}$ is not and becomes leading contribution. The dominant contribution in $d_{e}^{\phi W}$ comes from the diagrams involving the top and bottom loops, which amounts to [86, 134, 135]

$$
\frac{\left(d_{e}^{\phi W}\right)_{t / b}}{e} \simeq \frac{3 \alpha_{\mathrm{em}}\left|V_{t b}\right|^{2}}{128 \pi^{3} s_{W}^{2}} \frac{m_{t}}{m_{H^{ \pm}}^{2}} \operatorname{Im}\left(\rho_{e e} \rho_{t t}\right) J_{1}\left(\tau_{W H^{ \pm}}, \tau_{t H^{ \pm}}\right),
$$

where $m_{b}=0$ and $V_{t b}$ is the (33) element of the CKM matrix, which is close to one [1]. $J_{1}$ is the loop function listed in appendix A. In general, this contribution has the $\rho_{b b}$ dependence but vanishes in the case of $m_{b}=0$. Note that $\left(d_{e}^{\phi W}\right)_{t / b}$ is absent in the softly-broken $Z_{2}$ 2 HDMs. For one of the $\rho_{b b}$-EWBG parameter points, e.g., $\operatorname{Im}\left(\rho_{b b}\right)=-0.15$, one would get $\left(d_{e}^{\phi W}\right)_{t / b} \simeq 1.2 \times 10^{-29} e \mathrm{~cm}$ in the cancellation region specified by eq. (3.27), together with $m_{H^{ \pm}}=391 \mathrm{GeV}$ and $\rho_{t t}=0.5$, which slightly exceeds the ACME18 bound. Therefore, the allowed region is not exactly determined by the cancellation condition but it occurs in its vicinity, as we show in our numerical analysis conducted below. It should be noted that even though $\rho_{t t}$ is real in $\rho_{b b}$-EWBG, its magnitude can be constrained by the electron EDM due to the proportionality of $\rho_{t t} \operatorname{Im}\left(\rho_{e e}\right)$.

Now we move on to discuss the $C_{S}$ contribution. We estimate $C_{S}$ using the CPviolating 4 -fermion interactions between the quarks and electron defined as

$$
\mathcal{L}_{4 f}^{\mathrm{CPV}}=\sum_{q} C_{q e}(\bar{q} q)\left(\bar{e} i \gamma_{5} e\right)
$$

where $C_{q e}=\sum_{\phi=h, H, A} g_{\phi \bar{q} q}^{S} g_{\phi \bar{e} e}^{P} / m_{\phi}^{2}$ (explicit forms of $g_{\phi \bar{q} q}^{S}$ and $g_{\phi \bar{e} e}^{P}$ are shown in appendix A) With those, $C_{S}$ is estimated as [136]

$$
C_{S}=-2 v^{2}\left[6.3\left(C_{u e}+C_{d e}\right)+C_{s e} \frac{41 \mathrm{MeV}}{m_{s}}+C_{c e} \frac{79 \mathrm{MeV}}{m_{c}}+62 \mathrm{MeV}\left(\frac{C_{b e}}{m_{b}}+\frac{C_{t e}}{m_{t}}\right)\right] .
$$

Note that for $c_{\gamma} \ll 1$ and $m_{H} \simeq m_{A}, C_{q e}$ for up- and down-type quarks are, respectively, cast into the form [86]

$$
C_{u e} \simeq \frac{1}{2 m_{H}^{2}} \operatorname{Im}\left(\rho_{e e} \rho_{u u}\right), \quad C_{d e} \simeq \frac{1}{2 m_{H}^{2}} \operatorname{Im}\left(\rho_{e e} \rho_{d d}^{*}\right) .
$$

Therefore, the dependences of the CP-violating phases are the same as those of $\left(d_{e}^{\phi \gamma}\right)_{u}^{\text {extr }}$ and $\left(d_{e}^{\phi \gamma}\right)_{d}^{\text {extr }}$, respectively.

In our numerical analysis, we parametrize $\rho_{f f}$, except for $\rho_{t t}$, as $\operatorname{Re}\left(\rho_{e e}\right)=$ $-r\left(\lambda_{e} / \lambda_{b}\right) \operatorname{Re}\left(\rho_{b b}\right)$ and $\operatorname{Im}\left(\rho_{e e}\right)=-r\left(\lambda_{e} / \lambda_{b}\right) \operatorname{Im}\left(\rho_{b b}\right)$. Though the CP-violating phases in the first and second generations of $\rho^{F}$ matrices have nothing to do with $\rho_{b b}$-EWBG, we fix them through the above relations. However, the effects of the extra $\mathrm{CP}$ violation are too small to affect our cancellation mechanism in $\rho_{b b}$-EWBG.

In figure $4,\left|d_{\mathrm{ThO}}\right|$ and its details are shown as functions of $r$. We take $\mathrm{BP} a$ for the Higgs spectrum and set $c_{\gamma}=0.1, \operatorname{Re}\left(\rho_{b b}\right)=0, \operatorname{Im}\left(\rho_{b b}\right)=-0.15$ and $\rho_{t t}=0.5$ as an example 


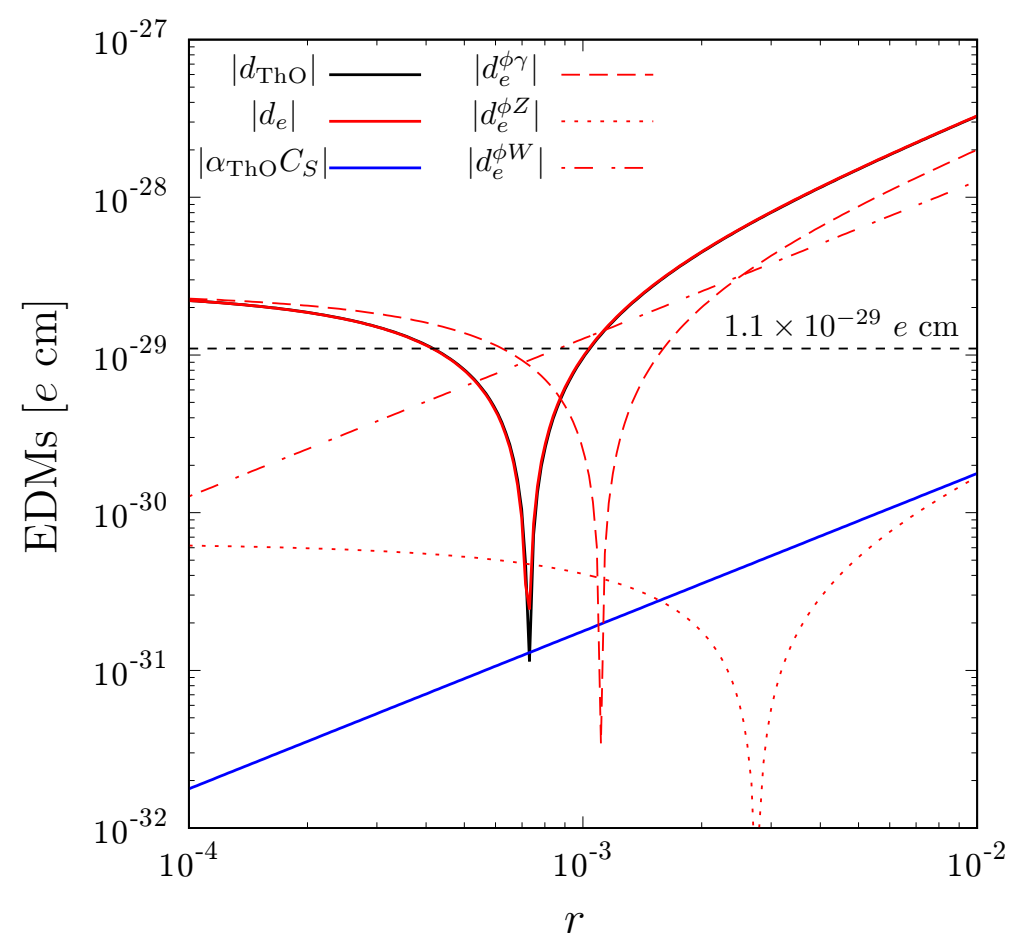

Figure 4. Details of EDMs as functions of $r$. We take BP $a$ for the Higgs spectrum and set $c_{\gamma}=0.1$, $\operatorname{Re}\left(\rho_{b b}\right)=0, \operatorname{Im}\left(\rho_{b b}\right)=-0.15$ and $\rho_{t t}=0.5$ as an example of the $\rho_{b b}$-EWBG scenario. Other $\rho_{f f}$ are fixed by $\operatorname{Re}\left(\rho_{e e}\right)=-r\left(\lambda_{e} / \lambda_{b}\right) \operatorname{Re}\left(\rho_{b b}\right)$ and $\operatorname{Im}\left(\rho_{e e}\right)=-r\left(\lambda_{e} / \lambda_{b}\right) \operatorname{Im}\left(\rho_{b b}\right)$. The ACME18 bound $\left(\left|d_{e}\right|<1.1 \times 10^{-29} e \mathrm{~cm}\right)$ is shown by the horizontal dotted line in black.

of the $\rho_{b b}$-EWBG scenario. As seen, the magnitude of $\alpha_{\mathrm{ThO}} C_{S}$ is much smaller than that of $d_{e}$, we thus can use the ACME18 bound of $\left|d_{e}\right|<1.1 \times 10^{-29} e \mathrm{~cm}$, which is represented by the horizontal dotted line in black, to constrain the parameter space. As discussed in eq. (3.27), the cancellation happens in $d_{e}^{\phi \gamma}$ at around $r \simeq 1 \times 10^{-3}$, which is the consequences of $\left(d_{e}^{\phi \gamma}\right)_{t}+\left(d_{e}^{\phi \gamma}\right)_{b}+\left(d_{e}^{\phi \gamma}\right)_{W} \simeq 0$. At this point, $d_{e}^{\phi W}$ becomes dominant and $\left|d_{e}\right|$ exceeds the ACME 18 bound. Nevertheless, the cancellation is still at work at around $r \simeq 0.7 \times 10^{-3}$. Similar to this case, we can always find cancellation regions in the cases of $\mathrm{BP} b$ and $\mathrm{BP} c$ as well, and thus conclude that $\rho_{b b}$-EWBG scenario is still consistent with the ACME18 bound. Note that here we set $c_{\gamma}=0.1$ while finding the constraints from ACME18 to illustrate $\operatorname{Im}\left(\rho_{b b}\right) \sim 0.15$ is still allowed for $\rho_{t t} \sim 0.5$ for all the three BPs. In the rest of the paper, however, we ignore the $c_{\gamma}$ dependence since it is insensitive to our collider study.

Here, we briefly discuss the EDMs of neutron and Mercury. Their current experimental values are respectively given by $[137,138]$

$$
\begin{aligned}
\left|d_{n}\right| & <1.8 \times 10^{-26} \text { e cm }(90 \% \text { C.L. }), \\
\left|d_{\mathrm{Hg}}\right| & <7.4 \times 10^{-30} \text { e cm }(95 \% \text { C.L. }) .
\end{aligned}
$$

On the theoretical side, the neutron EDM based on QCD sum rules is estimated as [139],

$$
d_{n}=-0.20 d_{u}+0.78 d_{d}+e\left(0.29 d_{u}^{C}+0.59 d_{d}^{C}\right) / g_{3},
$$


where $g_{3}$ is the $\mathrm{SU}(3)_{C}$ gauge coupling and $d_{q}^{C}$ are the quark chromo EDMs defined by the operator $\mathcal{L}_{\mathrm{CEDM}}=-(i / 2) d_{q}^{C} G^{\mu \nu} \bar{q} \sigma_{\mu \nu} \gamma_{5} q$ with $G^{\mu \nu}$ representing $\mathrm{SU}(3)_{C}$ field strength tensor. We note that even though the cancellation mechanism can work in $d_{n}$ as well, it does not occur at the cancellation point of $d_{e}$. Using the same input parameters as in figure 4 with $r=0.7 \times 10^{-3}$, we obtain $\left|d_{n}\right|=2.4 \times 10^{-29} e \mathrm{~cm}$, which is nearly 3 orders of magnitude below the current bound. For the mercury EDM, on the other hand, we estimate it using formulas in refs. [140-142] assuming $d_{\mathrm{Hg}}^{\mathrm{I}}$ defined in ref. [141] and find that $\left|d_{\mathrm{Hg}}\right|=$ $8.0 \times 10^{-31} e \mathrm{~cm}$, which is smaller than the current bound by about 1 order of magnitude.

We note in passing that a future measurement of the proton EDM could be another good prober of $\rho_{b b}$-EWBG. The experimental sensitivity of the proton EDM at IBS-CAPP [143] and BNL [144] is $\left|d_{p}\right| \sim 10^{-29} e \mathrm{~cm}$. As is the case of neutron EDM discussed above, the proton EDM can be estimated by use of the QCD sum rules as [139]

$$
d_{p}=0.78 d_{u}-0.20 d_{d}+e\left(-1.2 d_{u}^{C}-0.15 d_{d}^{C}\right) / g_{3}
$$

With this, it is found that $\left|d_{p}\right|=6.1 \times 10^{-29} e \mathrm{~cm}$ for the parameters used in figure 4 with $r=0.7 \times 10^{-3}$. Therefore, the future measurement of $d_{p}$ could access the $\rho_{b b}$-EWBG parameter space regardless of the $d_{e}$ cancellation.

\subsection{Direct search limits}

There exist several direct search limits from ATLAS and CMS that may restrict the parameter space of $\rho_{b b}$, even for $c_{\gamma}=0$ and $\rho_{t t}=0$. The coupling $\rho_{b b}$ receives several constraints from heavy Higgs boson searches at the LHC. In particular, refs. [145-149] are relevant to our study. We find that the most stringent constraint arises from CMS search involving heavy Higgs boson production in association with at least one $b$-jet and decaying into $b \bar{b}$ pair based on $13 \mathrm{TeV} 35.7 \mathrm{fb}^{-1}$ data [145]. The CMS search provides a model independent $95 \%$ CL upper limits on the $\sigma(p p \rightarrow b A / H+X) \cdot \mathcal{B}(A / H \rightarrow b \bar{b})$ in the mass range be-

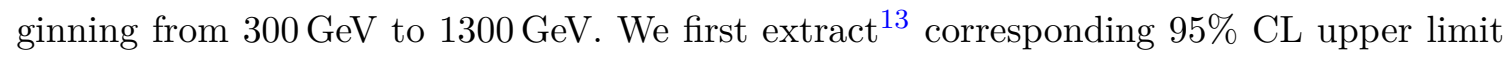
$\sigma(p p \rightarrow b A / H+X) \cdot \mathcal{B}(A / H \rightarrow b \bar{b})$ for our three BPs. Taking a reference $\left|\rho_{b b}\right|$ value, we then estimate the production cross sections of $p p \rightarrow b A / H+X$ at the leading order (LO) utilizing Monte Carlo event generator MadGraph5_aMC@NLO [150] (denoted as MadGraph5_aMC) with the default parton distribution function (PDF) NN23LO1 set [151] for the BPs. As the analysis does not veto additional activity in the event [145], we therefore include contributions from $g g \rightarrow b \bar{b} A / H$ along with $b g \rightarrow b A / H$ while estimating the cross sections. These cross sections are finally rescaled by $\left|\rho_{b b}\right|^{2} \times \mathcal{B}(A / H \rightarrow b \bar{b})$, assuming $\mathcal{B}(A / H \rightarrow b \bar{b})=100 \%$, to obtain the corresponding 95\% CL upper limits on $\left|\rho_{b b}\right|$. It is found that $\left|\rho_{b b}\right| \gtrsim 0.6$ is excluded for $\mathrm{BP} a$ at $95 \%$ CL and likewise, the regions where $\left|\rho_{b b}\right| \gtrsim$ 0.7 are ruled out for both $\mathrm{BP} b$ and $\mathrm{BP} c$. These upper limits are rather weak and would be further weakened by $\mathcal{B}(A \rightarrow Z h)$ and $\mathcal{B}(A / H \rightarrow t \bar{t})$. The limits are even weaker from a similar search performed by ATLAS [146]. We note that while estimating the upper limit

\footnotetext{
${ }^{13}$ To obtain the $95 \% \mathrm{CL} \sigma(p p \rightarrow b A / H+X) \cdot \mathcal{B}(A / H \rightarrow b \bar{b})$ upper limit for the three benchmark points BPI, BPII and BPIII, we digitized the figure of ref. [145]. The figure is available in http://cmsresults.web.cern.ch/cms-results/public-results/publications/HIG-16-018/ along with other auxiliary materials (a similar digitization strategy was followed in ref. [177]).
} 
on $\rho_{b b}$ we set all $\rho_{i j}=0$ for simplicity. In general, we remark that nonzero $\rho_{i j}$ would further alleviate these upper limits. Further, $\rho_{b b}$ coupling can induce $p p \rightarrow t(b) H^{ \pm}$process which is proportional to $V_{t b}$ (see eq. (2.7)). These processes are extensively searched by ATLAS [147] and CMS $[148,149]$ with $H^{+} / H^{-} \rightarrow t \bar{b} / \bar{t} b$ decays. We find that the constraints are weaker for all the three BPs, however, as we see below these searches would provide sensitive probe to $\rho_{t t}$. The effective model is implemented in the FeynRules 2.0 [152] framework.

We now turn to constraints on $\rho_{t t}$. As $\rho_{t t}$ can also induce $V_{t b}$, the searches $p p \rightarrow \bar{t}(b) H^{+}$ followed by $H^{+} \rightarrow t \bar{b}$ [147-149] would also be relevant. The ATLAS search [147] is based on $36 \mathrm{fb}^{-1} \sqrt{s}=13 \mathrm{TeV}$ dataset, which provides model independent 95\% CL upper limit on $\sigma\left(p p \rightarrow \bar{t} b H^{+}\right) \times \mathcal{B}\left(H^{+} \rightarrow t \bar{b}\right)$ from $m_{H^{ \pm}}=200 \mathrm{GeV}$ and $2 \mathrm{TeV}$. Similar searches are also performed by CMS based on $\sqrt{s}=13 \mathrm{TeV} 35.9 \mathrm{fb}^{-1}$ dataset [148, 149]. These searches provide 95\% CL upper limit on $\sigma\left(p p \rightarrow \bar{t} H^{+}\right) \times \mathcal{B}\left(H^{+} \rightarrow t \bar{b}\right)$ for $m_{H^{ \pm}}=200 \mathrm{GeV}$ and $3 \mathrm{TeV}$ in leptonic [148] and, combining leptonic and all-hadronic final states [149]. Like before, the nonvanishing $\rho_{t t}$ enhanced by $V_{t b}$ can induce such process, leading to stringent constraints. To find the constraints, as done before, we calculate the cross sections $\sigma(p p \rightarrow$ $\left.\bar{t} b H^{+}\right) \times\left(H^{+} \rightarrow t \bar{b}\right)$ at LO for a reference $\left|\rho_{t t}\right|$ for the three BPs via MadGraph5_aMC. These cross sections are then rescaled by $\left|\rho_{t t}\right|^{2} \times \mathcal{B}\left(H^{+} \rightarrow t \bar{b}\right)$ to get the corresponding $95 \%$ CL upper limits on $\left|\rho_{t t}\right|$. The extracted (footnote 13) 95\% CL upper limits from ATLAS search [147] on $\rho_{t t}$ for the three BPs are $\left|\rho_{t t}\right| \gtrsim 0.7,0.8$ and 1, respectively, while the limits from CMS [149] are much stronger, which read as $\left|\rho_{t t}\right| \gtrsim 0.6,0.61$ and 0.61 , respectively. We remark that the constraints from CMS search with leptonic final state [148] is mildly weaker than the search with combined leptonic and all-hadronic final states [149]. We also note that all the $\rho_{i j}$ except for $\rho_{t t}$ are assumed to be zero when extracting the upper limits for the sake of simplicity. Therefore if other $\rho_{i j}$ are turned on, the limits on $\rho_{t t}$ in general becomes weaker due to dilution from other branching ratios of $H^{ \pm}$.

The ATLAS [106] and CMS [107] search for heavy Higgs via $g g \rightarrow H / A \rightarrow t \bar{t}$ would also constrain $\rho_{t t}$. The ATLAS [106] result is based on $20.3 \mathrm{fb}^{-1}$ data at $8 \mathrm{TeV}$, which provides exclusion limits on $\tan \beta$ vs $m_{A}$ (or, $m_{H}$ ) in type-II 2 HDM framework starting from $m_{A}$ and $m_{H}=500 \mathrm{GeV}$. The CMS search is based on $35.9 \mathrm{fb}^{-1}$ data at $\sqrt{s}=13 \mathrm{TeV}$, which provides upper limit on coupling modifier (see ref. [107] for definition) the $m_{A}\left(m_{H}\right)$ from $400 \mathrm{GeV}$ to $750 \mathrm{GeV}$ based on different values of $\Gamma_{A} / m_{A}\left(\Gamma_{H} / m_{H}\right)$ ratios. Given the values of $m_{A}$ and $m_{H}$ of the BPs, ATLAS search can only constrain $\mathrm{BP} c$ via $g g \rightarrow H \rightarrow t \bar{t}$. Reinterpreting the ATLAS exclusion limit [106], we find that $\left|\rho_{t t}\right| \gtrsim 0.8$ is excluded for BP $c$ at 95\% CL. On the other hand, for CMS search [107], $g g \rightarrow A \rightarrow t \bar{t}$ can constrain $\left|\rho_{t t}\right|$ only for $\mathrm{BP} c$, whereas $g g \rightarrow H \rightarrow t \bar{t}$ constrains all three BPs. We find that CMS $g g \rightarrow A \rightarrow t \bar{t}$ search excludes the region of $\left|\rho_{t t}\right| \gtrsim 1$ (1.1) at $95 \%$ CL for $\mathrm{BP} c$ if $\Gamma_{A} / m_{A}=5 \%\left(\Gamma_{A} / m_{A}=10 \%\right)$. The $g g \rightarrow H \rightarrow t \bar{t}$ search places the constraints that $\left|\rho_{t t}\right| \gtrsim 1.6$ (2.1), 1.2 (1.4) and 1.2 (1.3) if $\Gamma_{H} / m_{H}=5 \%\left(\Gamma_{H} / m_{H}=10 \%\right)$ at $95 \% \mathrm{CL}$ for the three BPs respectively. We remark that these upper limits provided by both the collaborations assume that $m_{A}$ and $m_{H}$ are decoupled from each other. Although $m_{A}$ and $m_{H}$ are separated sufficiently, this is not the case for any of the BPs chosen, as can be seen from table 1. Therefore, the actual upper limits extracted here would be mildly stronger. 


\begin{tabular}{|c|c|c|c|}
\hline $\mathrm{BP}$ & $\mathcal{B}(A \rightarrow b \bar{b})$ & $\mathcal{B}(A \rightarrow Z h)$ & $\mathcal{B}(A \rightarrow t \bar{t})$ \\
\hline$a$ & 0.95 & 0.05 & - \\
$b$ & 0.89 & 0.11 & - \\
$c$ & 0.12 & 0.04 & 0.84 \\
\hline
\end{tabular}

Table 2. Branching ratios of $A$ for the benchmark points in table 1 with $\operatorname{Re}\left(\rho_{b b}\right)=0.0,\left|\operatorname{Im}\left(\rho_{b b}\right)\right|=$ 0.15 and $\left|\rho_{t t}\right|=0.5$.

Moreover, $\rho_{t t}$ would also receive constraint from CMS search for SM four-top production [153]. The search is performed with $13 \mathrm{TeV} 137 \mathrm{fb}^{-1}$ dataset and provides 95\% CL upper limits on $\sigma(p p \rightarrow t \bar{t} A / t \bar{t} H) \times \mathcal{B}(A / H \rightarrow t \bar{t})$ for $350 \mathrm{GeV} \leq m_{A / H} \leq 650 \mathrm{GeV}$. The search also includes contributions from $\sigma(p p \rightarrow t W A / H, t q A / H)$ followed by $A / H \rightarrow t \bar{t}$, which can also be induced by $\rho_{t t}$. To understand how strong the constraints could be, we generate these cross sections at LO by MadGraph5_aMC for a reference value of $\left|\rho_{t t}\right|$ setting all other $\rho_{i j}=0$, and then rescale simply by $\left|\rho_{t t}\right|^{2} \times \mathcal{B}(A / H \rightarrow t \bar{t})$. Again, given the masses of BPs, the $\sigma(p p \rightarrow t \bar{t} A) \times \mathcal{B}(A \rightarrow t \bar{t})$ search can constrain only BP $c$, for which $\left|\rho_{t t}\right| \gtrsim 0.8$ excluded at $95 \%$ CL. However, $\sigma(p p \rightarrow t \bar{t} H) \times \mathcal{B}(H \rightarrow t \bar{t})$ can constrain all three BPs, and we find that the regions of $\left|\rho_{t t}\right| \gtrsim 0.9,0.8$ and 1 are excluded at $95 \%$ CL, respectively, where $\mathcal{B}(A / H \rightarrow t \bar{t})=100 \%$ is assumed. However, it should be noted that the presence of $\mathcal{B}(A / H \rightarrow b \bar{b})$ and $\mathcal{B}(A \rightarrow Z h)$ would alleviate the limits. As in the case of $g g \rightarrow H / A \rightarrow t \bar{t}$, the search here also assumes that $m_{A}$ and $m_{H}$ are decoupled from each other. Therefore, one expects the limits to be mildly stronger for all the three BPs.

We finally conclude that for $\operatorname{Re}\left(\rho_{b b}\right) \sim 0, c_{\gamma}=0.1,\left|\operatorname{Im}\left(\rho_{b b}\right)\right| \sim 0.15$ and $\rho_{t t} \sim 0.5$ are well allowed by the current measurements for the mass spectrum under consideration. We take these values as representative values for our analysis with $\operatorname{Re}\left(\rho_{b b}\right)=0 .{ }^{14}$ Under the assumptions, i.e., setting all $\rho_{i j}=0$ except $\rho_{b b}$ and $\rho_{t t}$, the total decay width of $A$ can be nicely approximated as the sum of the partial widths of $A \rightarrow b \bar{b}$ and $A \rightarrow Z h$ for $\mathrm{BP} a$ and $\mathrm{BP} b$ and, $A \rightarrow b \bar{b}, A \rightarrow Z h$ and $A \rightarrow t \bar{t}$ for $\mathrm{BP} c$. The total decay widths of $A$ are $0.4 \mathrm{GeV}, 0.5 \mathrm{GeV}$ and $4.97 \mathrm{GeV}$ respectively for the three BPs with $\left|\operatorname{Im}\left(\rho_{b b}\right)\right| \sim 0.15$ and $\rho_{t t} \sim 0.5$. The corresponding branching ratios are presented in table 2. Note that when calculating decay widths and branching ratios of $A$, we neglect tiny loop induced decays such as $A \rightarrow \gamma \gamma, A \rightarrow Z \gamma$ etc.

\section{Collider signatures}

\subsection{The $b g \rightarrow b A \rightarrow b Z h$ process}

In this subsection we study the discovery potential of $b g \rightarrow b A \rightarrow b Z h$ process at $14 \mathrm{TeV}$ LHC. The process can be searched via $p p \rightarrow b A+X \rightarrow b Z h+X$ followed by $Z \rightarrow \ell^{+} \ell^{-}(\ell=$ $e, \mu)$ and $h \rightarrow b \bar{b}$, comprising three $b$-jets, same flavor opposite sign lepton pair (denoted

\footnotetext{
${ }^{14}$ As discussed before, both $\rho_{b b}$ and $\rho_{t t}$ can induce $p p \rightarrow t(b) H^{ \pm}$process, hence, the constraints from ref. [149] would become stronger if both the couplings are nonvanishing. However, we have checked that $\rho_{t t} \sim 0.5$ is allowed for $\left|\operatorname{Im}\left(\rho_{b b}\right)\right| \sim 0.15$ for all the three BPs.
} 

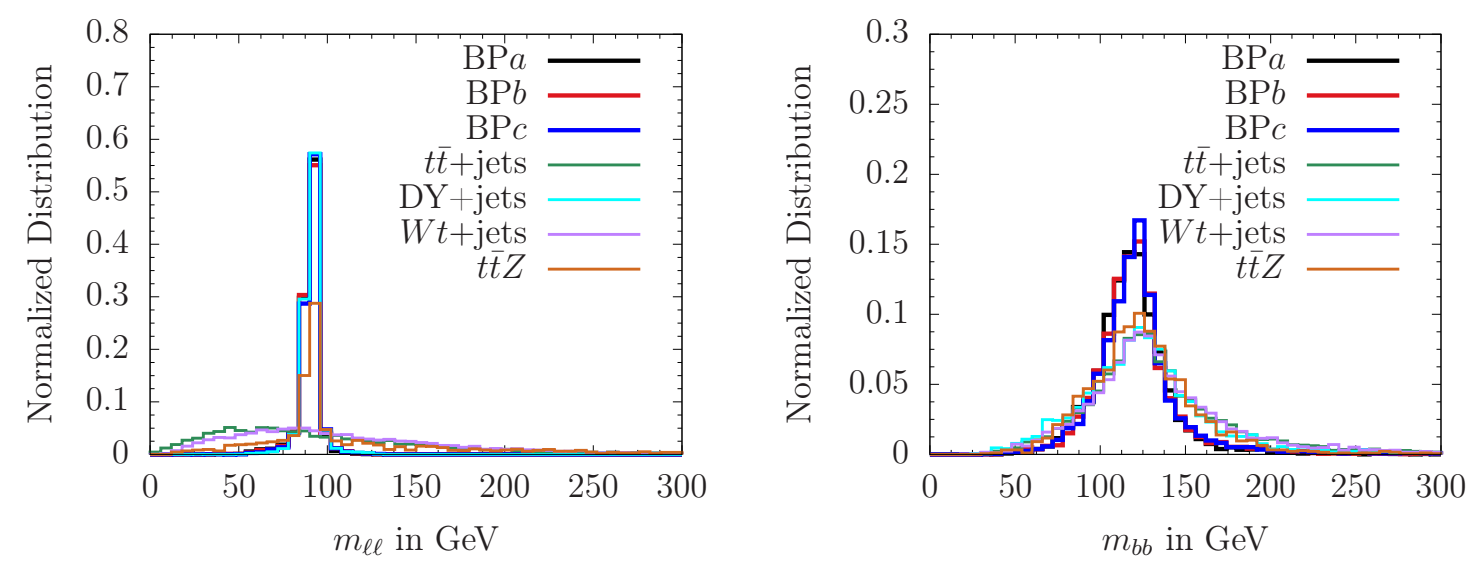

Figure 5. The normalized $m_{\ell \ell}$ (left) and $m_{b b}$ (right) distributions of the three BPs and leading backgrounds for the $b Z h$ process.

as the $b Z h$ process). There are several SM backgrounds for this final state topology. The dominant backgrounds are $t \bar{t}+$ jets, Drell-Yan + jets $(\mathrm{DY}+$ jets $), W t+$ jets, $t \bar{t} Z+$ jets, $t \bar{t} h$, $t Z+$ jets, with subdominant contributions arise from four-top $(4 t), t \bar{t} W, t W h, t W Z$ and $W Z+$ jets. Backgrounds from $W W+$ jets and $Z Z+$ jets are negligibly small and hence not included. Note that one can also search for $h \rightarrow \gamma \gamma$ or $h \rightarrow \tau \tau$; however, we do not find them as promising.

The signal and background event samples are generated in $p p$ collision with $\sqrt{s}=$ $14 \mathrm{TeV}$ CM energy at LO by MadGraph5_aMC with NN23LO1 PDF set as done before and then interfaced with Pythia 6.4 [154] for hadronization and showering and finally fed into Delphes 3.4.2 [155] for fast detector simulation adopting default ATLAS-based detector card. We adopt MLM scheme $[156,157]$ for matrix element and parton shower merging. Note that we have not included backgrounds from the fake and non-prompt sources in our analysis. Such backgrounds are not properly modeled in Monte Carlo simulations and requires data to estimate such contributions.

The LO $t \bar{t}+$ jets and $W t+$ jets cross sections are normalized to NNLO (next-to-next-to LO) with NNLL (next-to-next-to leading logarithmic) corrections by factors 1.84 [158] and 1.35 [159] respectively. We normalize the DY+jets background cross section to the NNLO QCD+NLO EW one by factor 1.27, which is obtained by utilizing FEWZ 3.1 [160, 161]. The LO $t \bar{t} Z, \bar{t} Z+$ jets, $t \bar{t} h, 4 t$ and $t \bar{t} W^{-}\left(t \bar{t} W^{+}\right)$cross sections are adjusted to NLO ones by $K$-factors 1.56 [162], 1.44 [150], 1.27 [163], 2.04 [150] and 1.35 (1.27) [164] respectively, but $t W Z$ and $t W h$ both are kept at LO. Finally, the background $W^{-} Z+$ jets is normalized to NNLO by factor 2.07 [165]. For simplicity we assume the same QCD correction factors for the conjugate processes $t Z j$ and $W^{+} Z+$ jets, while the signal cross sections are kept at LO.

To reduce backgrounds, we adopt following event selection criteria: each event should contain a same flavor opposite sign lepton pair and at least three $b$-tagged jets. The transverse momenta $\left(p_{T}\right)$ of the leading and subleading leptons should be $>28 \mathrm{GeV}$ and $>25 \mathrm{GeV}$ respectively, whereas $p_{T}$ for all three $b$-jets should be $>20 \mathrm{GeV}$. The pseudorapidity $(|\eta|)$ of the leptons and all three $b$-jets are required to be $<2.5$. The jets are 


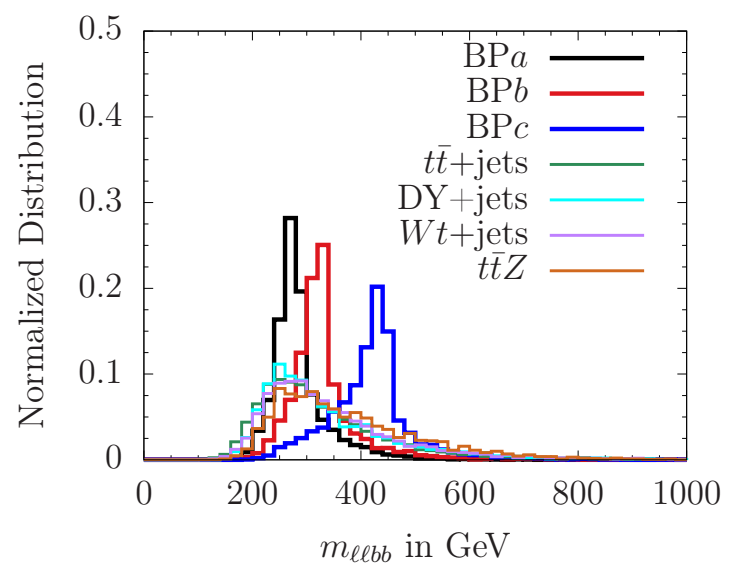

Figure 6. The normalized $m_{\ell \ell b b}$ distributions of the signal and leading backgrounds for the $b Z h$ process.

\begin{tabular}{|c|c|c|c|c|c|c|c|c|}
\hline BP & $t \bar{t}+$ jets & $D Y+$ jets & $W t+$ jets & $t \bar{t} Z$ & $t \bar{t} h$ & $t Z+$ jets & Others & $\begin{array}{c}\text { Total Bkg. } \\
(\mathrm{fb})\end{array}$ \\
\hline & & & & & & & & \\
$a$ & 0.178 & 0.533 & 0.226 & 0.023 & 0.008 & 0.007 & 0.003 & 0.978 \\
$b$ & 0.087 & 0.272 & 0.084 & 0.018 & 0.003 & 0.006 & 0.001 & 0.471 \\
$c$ & 0.016 & 0.094 & 0.022 & 0.008 & 0.0002 & 0.002 & 0.0004 & 0.143 \\
\hline
\end{tabular}

Table 3. The background cross sections (in fb) for the $b Z h$ process after selection cuts at $\sqrt{s}=$ $14 \mathrm{TeV}$ LHC. Here we added together subdominant backgrounds $4 t, t \bar{t} W, t W h, t W Z$ and $W Z+$ jets as "Others". The total background (Total Bkg.) yield is provided in last column.

\begin{tabular}{|c|c|c|}
\hline BP & $\begin{array}{c}\text { Signal } \\
(\mathrm{fb})\end{array}$ & $\begin{array}{c}\text { Significance }(\mathcal{Z}) \\
300(1000) \mathrm{fb}^{-1}\end{array}$ \\
\hline$a$ & 0.17 & $2.9(5.3)$ \\
$b$ & 0.228 & $5.4(9.8)$ \\
$c$ & 0.027 & $1.2(2.2)$ \\
\hline
\end{tabular}

Table 4. The signal cross sections after selection cuts and the corresponding significances of the $b Z h$ process with $300(1000) \mathrm{fb}^{-1}$ integrated luminosity.

reconstructed by anti- $k_{T}$ algorithm with radius parameter $R=0.4$. The separation $\Delta R$ between any two $b$-jets, a $b$-jet and a lepton and between two leptons should be $>0.4$. In order to reduce the $t \bar{t}+$ jets background, we veto events having missing transverse energy $\left(E_{T}^{\text {miss }}\right)>35 \mathrm{GeV}$. The invariant mass of the two same flavor opposite charge leptons $\left(m_{\ell \ell}\right)$ is needed to remain between $76<m_{\ell \ell}<100 \mathrm{GeV}$, i.e., the $Z$ boson mass window. We then apply invariant mass for two $b$-jets $m_{b b}$ in a event. As there are at least three $b$-jets in a event, more than one $m_{b b}$ combinations are possible; the one closest to $m_{h}$ is selected and 
required to remain within $\left|m_{h}-m_{b b}\right|<25 \mathrm{GeV}$. Further, we require the invariant mass $m_{\ell \ell b b}$ constructed from the two same flavor opposite charge leptons and $b$-jets combination that passes the $m_{b b}$ selection to be within $\left|m_{A}-m_{\ell \ell b b}\right|<50 \mathrm{GeV}$. The normalized $m_{\ell \ell}$ and $m_{b b}$ distributions before application of any selection cuts are presented in figure 5 while the same for $m_{\ell \ell b b}$ is shown in figure 6 . We adopt the $b$-tagging efficiency and $c$-and light-jets misidentification efficiencies of Delphes ATLAS based detector card. The background cross sections after selection cuts of the three benchmark points are summarized in table. 3 , while the signal cross sections along with their corresponding significances with the integrated luminosity $\mathcal{L}=300$ and $1000 \mathrm{fb}^{-1}$ are presented in table 4 . The statistical significances are estimated using $\mathcal{Z}=\sqrt{2[(S+B) \ln (1+S / B)-S]}[167]$, where $S$ and $B$ are the numbers of the signal and background events.

Let us take a closer look at table 4 . We find very promising the discovery potential with sufficiently large $S / B$ ratio, especially for $m_{A}<2 m_{t}$. The achievable significance for the $\mathrm{BP} a$ and $\mathrm{BP} b$ are $\sim 2.9 \sigma(\sim 5.3 \sigma)$ and $\sim 5.4 \sigma(\sim 9.8 \sigma)$ respectively with $300(1000) \mathrm{fb}^{-1}$ integrated luminosity. The $\mathrm{BP} c$ requires larger dataset due to fall in parton luminosity and suppression from $\mathcal{B}(A \rightarrow t \bar{t})$ decay and $\sim 2.2 \sigma$ is possible with $1000 \mathrm{fb}^{-1}$ but could reach up to $\sim 3.8 \sigma$ with the full high luminosity LHC (HL-LHC) dataset (3000 $\mathrm{fb}^{-1}$ integrated luminosity).

\subsection{The $b g \rightarrow b A \rightarrow b t \bar{t}$ process}

We now discuss the discovery potential of $p p \rightarrow b A+X \rightarrow b t \bar{t}+X$, followed by semileptonic decay of at least one top quark, constituting three $b$-jets, at least one charged lepton $(e$ and $\mu$ ) and missing transverse energy $\left(E_{T}^{\text {miss }}\right)$ signature, which we denote as $3 b 1 \ell$ signature. Note that $b g \rightarrow b A \rightarrow b t \bar{t}$ is only possible for $\mathrm{BP} c$ as $m_{A}<2 m_{t}$ but for $\mathrm{BP} a$ and $\mathrm{BP} b$ one can have $3 b 1 \ell$ signature via $p p \rightarrow b H+X \rightarrow b t \bar{t}+X$. However, such signatures will be mild for the former two BPs due to suppression from $\mathcal{B}(H \rightarrow A Z)$. Further, $b g \rightarrow \bar{t} H^{+} \rightarrow \bar{t} t \bar{b}$ process may also contribute to the same final state topologies, if at least one of the top decays semileptonically. Such contribution could be moderate for all three BPs. In our analysis, however, we neglect them for simplicity.

There exist several SM backgrounds. The dominant backgrounds are $t \bar{t}+\mathrm{jets}, t$ - and $s$ channel single-top $(t j), W t$, with subdominant backgrounds from $t \bar{t} h$ and, $t \bar{t} Z$ productions. Further, small contributions come from Drell-Yan+jets, $W+$ jets, four-top (4t), $t \bar{t} W, t W h$, which are collectively denoted as "Others". We do not include backgrounds originating from non-prompt and fake sources. These backgrounds are not properly modeled in Monte Carlo event generators and one requires data to estimate such contributions.

Here we follow the same event generation procedure for signal and backgrounds as in previous subsections, i.e., via MadGraph5_aMC followed by hadronization and showering in Pythia and with Delphes ATLAS based detector card for fast detector simulation. The LO $t \bar{t}+$ jets background cross section is normalized up to the NNLO by a factor of 1.84 while $t$ - and $s$-channel single-top cross sections are normalized by factors of 1.2 and 1.47 , respectively [166]. The LO $W t+$ jets background is normalized to the NLO cross section by a factor of 1.35 , whereas the subdominant $t \bar{t} h$ and $t \bar{t} Z$ are corrected to corresponding NLO ones by factors of 1.27 and 1.56 respectively. The DY+jets background is normalized 

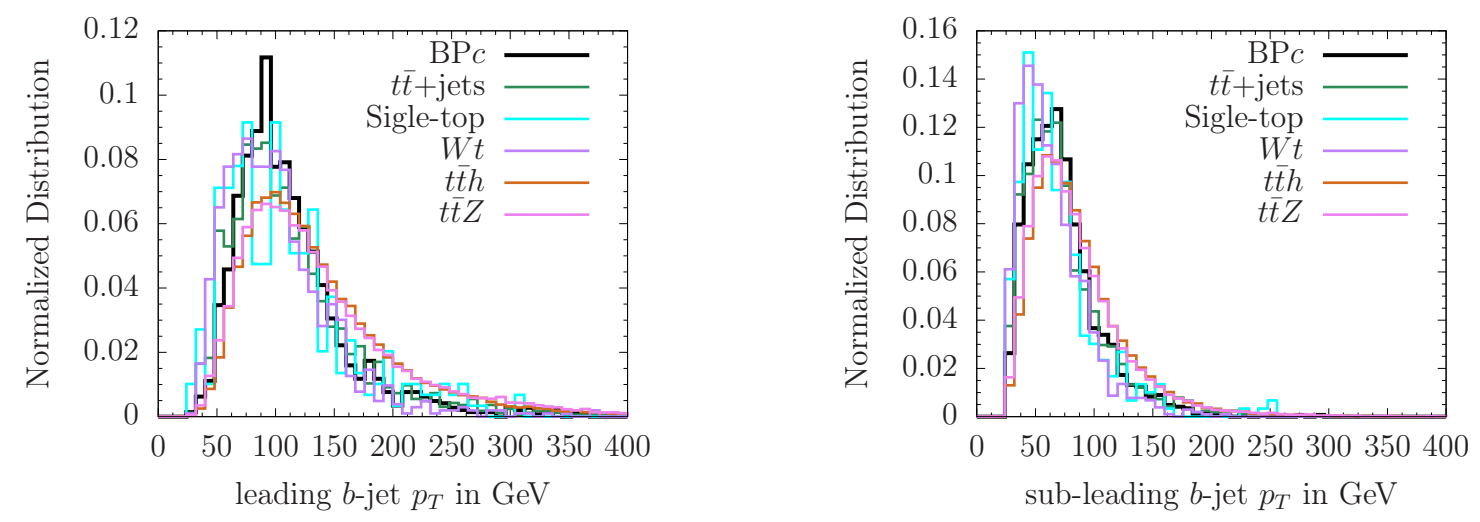

Figure 7. The normalized $p_{T}$ distributions of the leading and subleading $b$-jets for the signal $(\mathrm{BP} c)$ and the leading backgrounds.
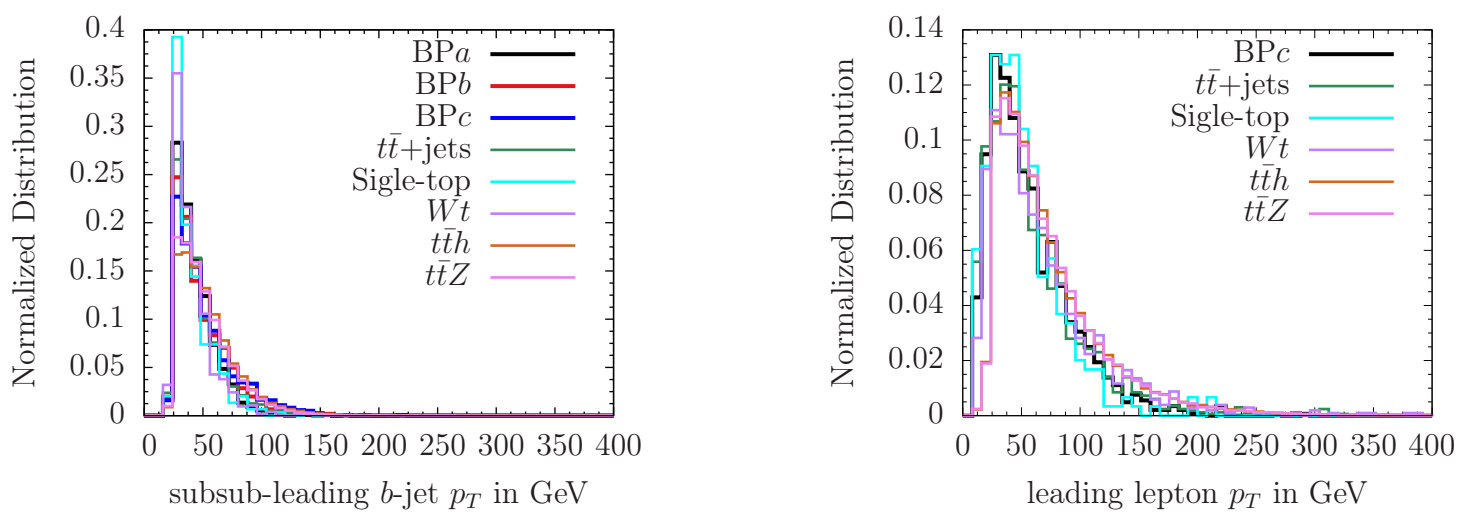

Figure 8. The normalized $p_{T}$ distributions of the subsubleading $b$-jet (right) and leading lepton (left) for the signal $(\mathrm{BP} c)$ and leading backgrounds.

to NNLO cross sections by factor of 1.27 . Finally, the LO cross sections $4 t$ and $t \bar{t} W$ are adjusted to the NLO ones by factors of 2.04 and 1.35, respectively. The $t W h$ and $W+$ jets background are kept at LO. For simplicity we assume the correction factors for the charge conjugate processes to be the same. We remark that the signal cross sections for all the three BPs are kept at LO.

The events are selected in a way such that they should contain at least one charged lepton $\left(e\right.$ and $\mu$ ), at least three $b$-tagged and some $E_{T}^{\text {miss }}$. The normalized transverse momentum $\left(p_{T}\right)$ distributions of the leading and subleading $b$-jets for the signal and leading backgrounds are presented in figure 7 . The $p_{T}$ distributions for the subsubleading $b$-jet and leading lepton are plotted in figure 8 , while the normalized $E_{T}^{\text {miss }}$ and $H_{T}$ (i.e., the scalar sum of $p_{T}$ of the leading charged lepton and the three leading $b$-jets) distributions are shown in figure 9 . To reduce backgrounds we apply the following event selection cuts. $p_{T}$ of all three $b$-jets should be $p_{T}>20 \mathrm{GeV}$, whereas that of the leading lepton should be $p_{T}>25 \mathrm{GeV}$. The absolute value of pseudo-rapidity $(|\eta|)$ of all three $b$-jets and lepton should be less than 2.5. The minimum separation $(\Delta R)$ between the lepton and any $b$-jet as well 

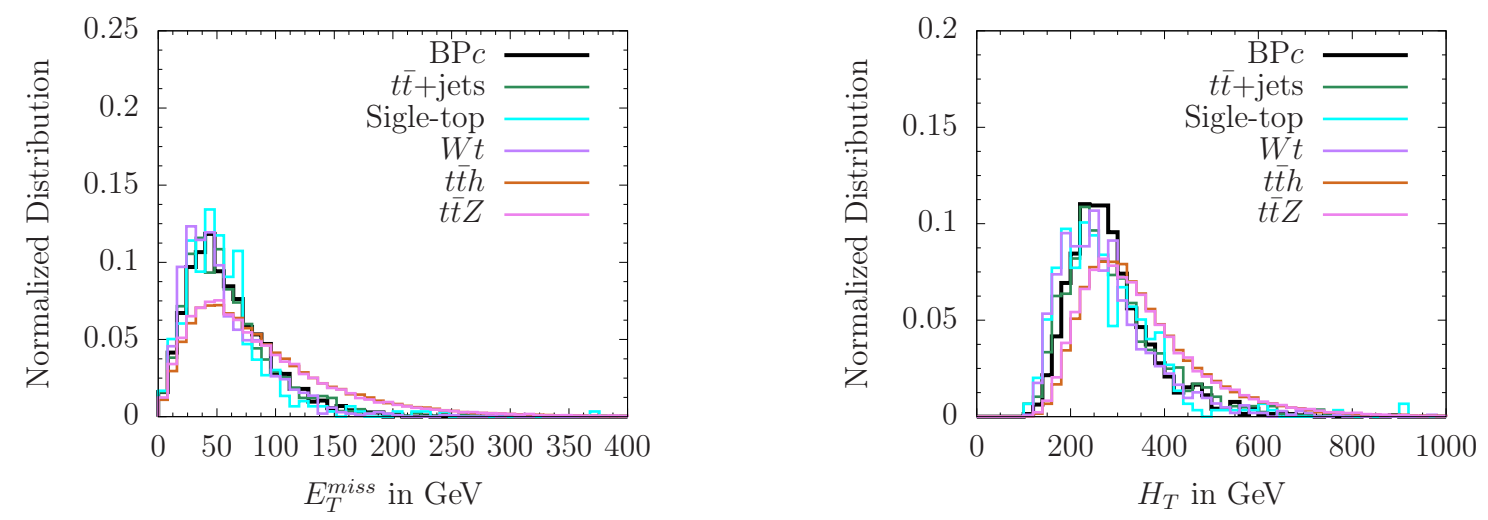

Figure 9. The normalized missing energy $E_{T}^{\text {miss }}$ (left) and $H_{T}$ (right) for the signal $(\mathrm{BP} c)$ and leading backgrounds.

\begin{tabular}{|c|c|c|c|c|c|c|c|c|}
\hline BP & Signal & $t \bar{t}+$ jets & Single-top & $W t+$ jets & $t \bar{t} h$ & $t \bar{t} Z$ & Others & $\begin{array}{c}\text { Total Bkg. } \\
(\mathrm{fb})\end{array}$ \\
\hline $\mathrm{BP} c$ & 9.27 & 3953.49 & 98.93 & 77.93 & 10.72 & 4.13 & 30.85 & 4176.05 \\
\hline
\end{tabular}

Table 5. The cross sections (in units of $\mathrm{fb}$ ) of the signal $(\mathrm{BP} c)$ and different background components for $3 b 1 \ell$ process after selection cuts at $\sqrt{s}=14 \mathrm{TeV}$.

as that between any two $b$-jets are required to be greater than 0.4. The $E_{T}^{\mathrm{miss}}$ in each event should be larger than $35 \mathrm{GeV}$. Note that in our exploratory study we do not optimize the selection cuts such as $p_{T}, \eta, E_{T}^{\text {miss }}$ and $H_{T}$ for simplicity. The signal and total background cross sections along with different components after the selection cuts are shown in table 5 .

The corresponding significance $\mathcal{Z}$ for the integrated luminosities $300(3000) \mathrm{fb}^{-1}$ for the $\mathrm{BP} c$ is $\sim 3.5 \sigma(\sim 7.9 \sigma)$. For the $\mathrm{BP} a$ and $\mathrm{BP} b$ from $p p \rightarrow b H+X \rightarrow b t \bar{t}+X$ are $\sim 2.4 \sigma$ and $\sim 2.8 \sigma$ respectively for $3000 \mathrm{fb}^{-1}$. Note that here we have not considered the systematic uncertainties associated with the backgrounds, which could be considerable in particular for the largest $t \bar{t}+$ jets backgrounds. As $S \ll B$ for the $3 b 1 \ell$ process, in the presence of systematic uncertainties the $\mathcal{Z}$ formula simply becomes $\mathcal{Z} \approx S / \sqrt{B+\sigma_{B}^{2}}$. The $\sigma_{B}$ denotes the systematic uncertainty that depends on the factor between control sample and the background in the signal region. The value of $\sigma_{B}$ is very much analysis ${ }^{15}$ dependent. Here we assume that the systematic uncertainty arise only from $t \bar{t}+$ jets backgrounds for simplicity and neglect the same for other subdominant backgrounds and take two different for $\sigma_{B}$ for illustration. E.g., if $\sigma_{B}=\sqrt{B}$ the significance of BP $c$ reduces to $\sim 5.5 \sigma$ whereas for $\sigma_{B}=0.1 B$ the significances goes below $1 \sigma$ with HL-LHC dataset. Similarly, for $\sigma_{B}=\sqrt{B}$ the significances of $\mathrm{BP} a$ and $\mathrm{BP} b$ from $p p \rightarrow b H+X \rightarrow b t \bar{t}+X$ reduces to $\sim 1.7 \sigma$ and $\sim 2 \sigma$ respectively but much below $1 \sigma$ if $\sigma_{B}=0.1 B$. Therefore we remark that the $3 b 1 \ell$ process is promising, but one needs precise understanding of the background systematics.

\footnotetext{
${ }^{15}$ We thank Efe Yazgan and K.-F. Chen for clarifying this point. We also thank the anonymous reviewer for bringing this issue to our attention.
} 


\begin{tabular}{|c|c|c|c|c|c|c|}
\hline BP & Signal & $t \bar{t}+$ jets & Single-top & $W t+$ jets & Others & Total Bkg. (fb) \\
\hline & & & & & & \\
$\mathrm{BP} a$ & 0.2 & 229.2 & 4.5 & 2.8 & 7.8 & 244.3 \\
$\mathrm{BP} b$ & 0.14 & 204.9 & 4.0 & 2.3 & 8.2 & 219.4 \\
$\mathrm{BP} c$ & 0.01 & 157.8 & 2.7 & 1.9 & 5.3 & 167.7 \\
\hline
\end{tabular}

Table 6. The cross sections (in units of $\mathrm{fb}$ ) of three BPs and the different background components for the $4 b 1 \ell$ process after selection cuts at $\sqrt{s}=14 \mathrm{TeV}$.

\subsection{The $g g \rightarrow t \bar{t} A \rightarrow t \bar{t} b \bar{b}$ process}

We now briefly discuss the discovery potential of $g g \rightarrow t \bar{t} A \rightarrow t \bar{t} b \bar{b}$ process. The process can in principle probe the parameter space for $\rho_{b b}$-EWBG mechanism. We search this process via $p p \rightarrow t \bar{t} A+X \rightarrow t \bar{t} b \bar{b}+X$, followed by at least one top quark decaying semileptonically i.e., with four $b$-jets, at least one charged lepton and $E_{T}^{\text {miss }}$ signature. The final state topology receives mild contributions from inclusive $p p \rightarrow W t A+X$ and $p p \rightarrow t j A+X$ processes. As we show below, the signature is not promising as opposed to $b g \rightarrow b A \rightarrow b t \bar{t}$ process.

We generate events at $\mathrm{LO}$ as in $3 b 1 \ell$ process, i.e., via MadGraph5_aMC followed by hadronization and showering in Pythia and finally incorporate the detector effects of Delphes ATLAS based detector card. The dominant backgrounds arise from the $t \bar{t}+$ jets, Single-top and $W t+$ jets, whereas $t \bar{t} h, t \bar{t} Z$ and $4 t$ constitute subdominant backgrounds. We assume the same QCD corrections factor as in $3 b 1 \ell$ process for simplicity.

To reduce the background, we use the following event selection cuts. The events are selected so that they contain at least one lepton $(e$ and $\mu)$, at least four jets with at least four are $b$-tagged and some missing $E_{T}^{\text {miss }}$ (denoted as $4 b 1 \ell$ process). The lepton is required to have $p_{T}>25 \mathrm{GeV}$ and $|\eta|<2.5$. For any jet in the event $p_{T}>20 \mathrm{GeV}$ and $|\eta|<2.5$. $E_{T}^{\text {miss }}$ in each event is required to be greater than $35 \mathrm{GeV}$. The separation $\Delta R$ between any two jets as well as that between a jet and a lepton should be larger than 0.4. Finally, we construct all possible combinations of the invariant mass $m_{j j}$ from the four leading jets and demand that the one closest to $m_{A}$ should lie between $\left|m_{A}-m_{j j}\right|<50 \mathrm{GeV}$. The impact of these cuts on the signal and background processes are summarized in table 6 .

We find that the achievable significance for all three BPs of the $g g \rightarrow t \bar{t} A \rightarrow t \bar{t} b \bar{b}$ process are below $\sim 1 \sigma$ with $3000 \mathrm{fb}^{-1}$ integrated luminosity, which is rather low. This means that no meaningful constraints can be extracted unless both ATLAS and CMS data are added. It should be remarked that since we use the same QCD correction factors for the backgrounds as in $b g \rightarrow b A \rightarrow b t \bar{t}$ process, there are greater uncertainties in these cross sections.

Before closing this section, we discuss the impact of the other $\rho_{i j}$ couplings. So far, we have set all $\rho_{i j}=0$ except $\rho_{b b}$ and $\rho_{t t}$. Presence of the other $\rho_{i j}$ couplings open up other decay modes of $A$, which in principle may reduce the achievable significances summarized in previous subsections. For instance, if $\rho_{\tau \tau} \sim \lambda_{\tau}$, it would induce $A \rightarrow \tau^{+} \tau^{-}$decay. However, 
the significances remain practically same for all the three BPs. Moreover, $\rho_{t c} \sim 0.3-0.4$ is still allowed by current data and would induce $c g \rightarrow t A / t H \rightarrow t t \bar{c}$ (same-sign top) [93, 168] (see also refs. $[169,170]^{16}$ ) and $c g \rightarrow t A / t H \rightarrow t t \bar{c}$ (triple-top) signature, which might emerge in the Run-3 of LHC.

\section{Discussion and summary}

We have analyzed the available constraints and prospect of probing EWBG driven by the extra bottom Yukawa coupling $\rho_{b b}$ at the ongoing and future experiments. The parameter space receives meaningful constraints from $h$ boson coupling measurements, $\mathcal{B}\left(B \rightarrow X_{s} \gamma\right)$, $\Delta \mathcal{A}_{\mathrm{CP}}$ of $B \rightarrow X_{s} \gamma$, electron EDM measurement and heavy Higgs searches at the LHC. We primarily focused on sub- $\mathrm{TeV} m_{A}, m_{H}$ and $m_{H^{ \pm}}$with mixing angle $c_{\gamma} \sim 0.1$, which is required by EWBG $[15-77,85]$. The constraints would be improved, e.g., on $c_{\gamma}, \rho_{b b}$ and $\rho_{t t}$ at the HL-LHC $[85,174]$ if no discovery is made. This would allow us to probe even larger part of the parameter space of $\rho_{b b}$-EWBG.

Taking three benchmark points for illustration, two below $2 m_{t}$ threshold and one above, we have shown that a discovery is possible at the LHC via $\rho_{b b}$ induced $b g \rightarrow b A \rightarrow$ $b Z h$ process for $m_{A}<2 m_{t}$. We find that the process may emerge in the Run 3 of LHC if $250 \mathrm{GeV} \lesssim m_{A} \lesssim 350 \mathrm{GeV}$. With a simple rescaling of the significances in table 4 , we find that $\left|\operatorname{Im}\left(\rho_{b b}\right)\right| \gtrsim 0.05$ and $\gtrsim 0.04$ can be excluded for $\mathrm{BP} a$ and $\mathrm{BP} b$ with full HLLHC dataset. Those are below the nominal value $\left|\operatorname{Im}\left(\rho_{b b}\right)\right| \gtrsim 0.058$ required EWBG. For $m_{A}>2 m_{t}$, a discovery may happen via $b g \rightarrow b A \rightarrow b t \bar{t}$. Note that one may also have the $b g \rightarrow b H \rightarrow b h h$ process, which could be sensitive if $H$ is lighter than $A$ and $H^{ \pm}$. The process is being studied elsewhere. A discovery of the $b g \rightarrow b A \rightarrow b t \bar{t}$ process is possible via $3 b 1 \ell$ signature if $m_{A} \sim 450 \mathrm{GeV}$ and $\rho_{t t} \sim 0.5$ but requires controlling of the systematics of $t \bar{t}$-jets background. Additionally, we have also investigated the potential for $g g \rightarrow t \bar{t} A \rightarrow t \bar{t} b \bar{b}$ process but find it below the sensitivity even at the HL-LHC.

In principle the $p p \rightarrow b A+X$ process can also be induced by $\rho_{b d}, \rho_{d b}, \rho_{b s}$ and $\rho_{s b}$ at the LHC. However, due to severe constraints arising from $B_{d}$ and $B_{s}$ mixings [176] their impacts are typically inconsequential. In addition if the charm quark gets misidentified as $b$-jet, a sizable $\rho_{c c}$ can also mimic similar signature in $p p$ collision via $c g \rightarrow c A \rightarrow c t \bar{t}$ process. We remark that such possibilities can be disentangled by the simultaneous application of $b$ and $c$-tagging on the final state topologies [177].

Although the discovery is possible at the LHC, to attribute it to $\rho_{b b}$-EWBG mechanism is beyond the scope of LHC as information of the CP-violating phase of $\rho_{b b}$ is lost in $p p$ collision. In this regard, $\Delta \mathcal{A}_{\mathrm{CP}}$ of $\mathcal{B}\left(B \rightarrow X_{s} \gamma\right)$ would provide very sensitive probe for the $\operatorname{Im}\left(\rho_{b b}\right)$ even though the observable has uncertainties associated with the hadronic parameter $\tilde{\Lambda}_{78}$. While finding the constraints in figure 2 , we assumed $\tilde{\Lambda}_{78}=89 \mathrm{MeV}$, which is the average of $17 \mathrm{MeV} \leq \tilde{\Lambda}_{78} \leq 190 \mathrm{MeV}$ [130]. However, if $\tilde{\Lambda}_{78}$ is taken as its upper range, the constraint becomes much severe for $\operatorname{Im}\left(\rho_{b b}\right)$. Furthermore, on the experimental side, projected Belle II accuracy of $\Delta \mathcal{A}_{\mathrm{CP}}$ measurement is $\sim 5 \%$ [175]. Therefore, more precise estimation of $\tilde{\Lambda}_{78}$ together with Belle II measurement can stringently probe the parameter

\footnotetext{
${ }^{16}$ Without detailed studies, the process was also discussed by $[171,172]$ and ref. [170]. See also ref. [173].
} 
space of $\operatorname{Im}\left(\rho_{b b}\right)$ unless the CP-violating phases of $\rho_{t t}$ and $\rho_{b b}$ are aligned [86] in which $\Delta \mathcal{A}_{\mathrm{CP}}=0$. In such a case, measurements of EDMs play a pivotal role in probing $\operatorname{Im}\left(\rho_{b b}\right)$.

The unprecedented electron EDM constraint set by ACME Collaboration in 2018 reduces most EWBG scenarios to despair. We updated our previous analysis done in ref. [85] including all the relevant Barr-Zee diagrams. Because of the significant contributions arising from the diagrams involving $\rho_{e e}$, the cancellation mechanism can be effective. It was found that the electron EDM cancellation in $\rho_{b b}$-EWBG belongs to the unstructured cancellation category in which the diagonal hierarchical structures of $\rho_{i j}^{F}$ are much different from those of the SM Yukawa couplings, which is in stark contrast to the case in $\rho_{t t}$-EWBG that can accommodate the structured cancellation [86]. Nonetheless, the viable parameter space of $\rho_{b b}$-EWBG still exists. Besides the extreme fine tuning of the parameters, $\rho_{b b}$-EWBG would be confirmed or ruled out if the electron EDM is improved down to $\sim 10^{-30} \mathrm{e} \mathrm{cm}$ level. Moreover, as discussed in section 3.2, the future measurement of the proton EDM could play a complementary role in probing $\rho_{b b}$-EWBG.

So far we have not discussed the uncertainties. As a first estimate, uncertainties arising from factorization scale $\left(\mu_{F}\right)$ and renormalization scale $\left(\mu_{R}\right)$ dependences are not included in our LO cross section estimations. In general, the LO $b g \rightarrow b A / b H$ processes have $\sim 25-30 \%$ scale uncertainties for $m_{A / H} \sim(300-400) \mathrm{GeV}$ if bottom quark with $p_{T}>(15-$ $30) \mathrm{GeV}$ and, $|\eta|<2.5$ [178] (see also [179-181]). It has been found that [182] the LO cross sections calculated with LO PDF set CTEQ6L1 [183] have relatively higher factorization scale dependence. Therefore, we remark that the LO cross sections in our analysis, which we estimated with LO NN23LO1 PDF set, might have same level of uncertainties. It has also been found that [182] for $\mu_{F} \approx m_{A}$ (or $m_{H}$ ) the corrections to the LO cross sections could be large negative $(\sim-70 \%)$, whereas for the choice of $\mu_{F} \approx m_{A} / 4$ (or $m_{H} / 4$ ) the corrections are mild; which indicates that the $\mu_{F} \approx m_{A} / 4$ is the relevant factorization scale. Furthermore, the cross section uncertainties from factorization scale and renormalization choices are found to be particularly small at $\mu_{R}=m_{A}$ and if varied from $\mu_{R}=m_{A} / 2$ to $\mu_{R}=2 m_{A}$, along with $\mu_{F}=m_{A} / 4$ and varied from $\mu_{F}=m_{A} / 8$ to $\mu_{F}=m_{A} / 2$ [182]. In addition, our analysis does not include PDF uncertainties, which could be in general significant for any bottom-quark initiated process as discussed, e.g., in ref. [184]. Detailed discussions on different PDFs and associated uncertainties for the LHC are summarized in ref. [185]. These typically would induce some uncertainties in our results. We leave out the detailed estimation of these uncertainties for future work.

In summary, we have explored the possibility of electroweak baryogenesis induced by the extra bottom Yukawa coupling $\rho_{b b}$ via direct and indirect signatures at the collider experiments. We find that the discovery is possible at the high luminosity LHC if $250 \mathrm{GeV} \lesssim m_{A} \lesssim 350 \mathrm{GeV}$. We also find that heavier mass ranges can also be probed via $b g \rightarrow b A \rightarrow b t \bar{t}$ but the process is associated with larger uncertainties. While LHC can indeed discover the process, however, the information of the CP-violating phase of $\rho_{b b}$ can only be probed via $\Delta \mathcal{A}_{\mathrm{CP}}$ of $\mathcal{B}\left(B \rightarrow X_{s} \gamma\right)$ or the EDM measurements of the electron, neutron and mercury though the latter two have the less probing power to date. For completeness we also studied $g g \rightarrow t \bar{t} A \rightarrow t \bar{t} b \bar{b}$ process and found that it is not promising. In conclusion, together with the electron EDM measurement and/or $\Delta \mathcal{A}_{\mathrm{CP}}$ of $\mathcal{B}\left(B \rightarrow X_{s} \gamma\right)$ 
decay, the discovery of $b g \rightarrow b A \rightarrow b t \bar{t}$ process may help us to understand the mechanism behind the observed matter-antimatter asymmetry of the Universe.

\section{Acknowledgments}

TM thanks Osaka University and Prof. Shiniya Kanemura for affiliation. TM was supposed to join Osaka University as a postdoctoral fellow in April but delayed due to travel restrictions related to ongoing pandemic. TM also thanks National Taiwan University and Prof. Wei-Shu Hou for temporary visiting position with grant number MOST 106-2112M-002-015-MY3.

\section{A EDMs}

For the EDM calculations, the following parametrization is also useful.

$$
\mathcal{L}_{\phi \bar{f} f}=-\phi \bar{f}\left(g_{\phi \bar{f} f}^{S}+i \gamma_{5} g_{\phi \bar{f} f}^{P}\right) f
$$

where $\phi=h, H, A$ and

$$
\begin{array}{rlrl}
g_{h \bar{f} f}^{S} & =\frac{1}{\sqrt{2}}\left[\lambda_{f} s_{\gamma}+\operatorname{Re} \rho_{f f} c_{\gamma}\right], & g_{h \bar{f} f}^{P} & =\frac{1}{\sqrt{2}} \operatorname{Im} \rho_{f f} c_{\gamma}, \\
g_{H \bar{f} f}^{S} & =\frac{1}{\sqrt{2}}\left[\lambda_{f} c_{\gamma}-\operatorname{Re} \rho_{f f} s_{\gamma}\right], & & g_{H \bar{f} f}^{P}=-\frac{1}{\sqrt{2}} \operatorname{Im} \rho_{f f} s_{\gamma}, \\
g_{A \bar{f} f}^{S} & = \pm \frac{1}{\sqrt{2}} \operatorname{Im} \rho_{f f}, & g_{A \bar{f} f}^{P}=\mp \frac{1}{\sqrt{2}} \operatorname{Re} \rho_{f f},
\end{array}
$$

where the upper sign is for up-type fermions and the lower for down-type fermions in eq. (A.4).

Here we list the loop functions appearing in the EDM calculations in section 3.2.

$$
\begin{gathered}
f(\tau)=\frac{\tau}{2} \int_{0}^{1} d x \frac{1-2 x(1-x)}{x(1-x)-\tau} \ln \left(\frac{x(1-x)}{\tau}\right), \\
g(\tau)=\frac{\tau}{2} \int_{0}^{1} d x \frac{1}{x(1-x)-\tau} \ln \left(\frac{x(1-x)}{\tau}\right), \\
\mathcal{J}_{W}^{V}\left(m_{\phi}\right)=\frac{2 m_{W}^{2}}{m_{\phi}^{2}-m_{V}^{2}}\left[-\frac{1}{4}\left\{\left(6-\frac{m_{V}^{2}}{m_{W}^{2}}\right)+\left(1-\frac{m_{V}^{2}}{2 m_{W}^{2}}\right) \frac{m_{\phi}^{2}}{m_{W}^{2}}\right\}\right. \\
\times\left(I_{1}\left(m_{W}, m_{\phi}\right)-I_{1}\left(m_{W}, m_{V}\right)\right) \\
+\left\{\left(-4+\frac{m_{V}^{2}}{m_{W}^{2}}\right)+\frac{1}{4}\left(6-\frac{m_{V}^{2}}{m_{W}^{2}}\right)+\frac{1}{4}\left(1-\frac{m_{V}^{2}}{2 m_{W}^{2}}\right) \frac{m_{\phi}^{2}}{m_{W}^{2}}\right\} \\
\left.\times\left(I_{2}\left(m_{W}, m_{\phi}\right)-I_{2}\left(m_{W}, m_{V}\right)\right)\right], \\
J_{1}\left(\tau_{W H^{ \pm}}, \tau_{t H^{ \pm}}\right)=\int_{0}^{1} \frac{d x}{x}(2-x)\left[Q_{t}(1-x) J\left(\tau_{W H^{ \pm}}, \frac{\tau_{t H^{ \pm}}}{x}\right)+Q_{b} x J\left(\tau_{W H^{ \pm}}, \frac{\tau_{t H^{ \pm}}}{x}\right)\right],
\end{gathered}
$$


where $\tau_{i j}=m_{i}^{2} / m_{j}^{2}, Q_{t}=2 / 3, Q_{b}=-1 / 3$ and

$$
\begin{aligned}
I_{1}\left(m_{1}, m_{2}\right) & =-2 \frac{m_{2}^{2}}{m_{1}^{2}} f\left(\frac{m_{1}^{2}}{m_{2}^{2}}\right), \quad I_{2}\left(m_{1}, m_{2}\right)=-2 \frac{m_{2}^{2}}{m_{1}^{2}} g\left(\frac{m_{1}^{2}}{m_{2}^{2}}\right), \\
J(a, b) & =\frac{1}{a-b}\left[\frac{a}{a-1} \ln a-\frac{b}{b-1} \ln b\right] .
\end{aligned}
$$

Open Access. This article is distributed under the terms of the Creative Commons Attribution License (CC-BY 4.0), which permits any use, distribution and reproduction in any medium, provided the original author(s) and source are credited.

\section{References}

[1] Particle Data Group collaboration, Review of particle physics, Phys. Rev. D 98 (2018) 030001 [INSPIRE].

[2] A.D. Sakharov, Violation of CP invariance, C asymmetry and baryon asymmetry of the universe, Sov. Phys. Usp. 34 (1991) 392 [Pisma Zh. Eksp. Teor. Fiz. 5 (1967) 32] [JETP Lett. 5 (1967) 24] [Usp. Fiz. Nauk 161 (1991) 61] [INSPIRE].

[3] ATLAS collaboration, Observation of a new particle in the search for the Standard Model Higgs boson with the ATLAS detector at the LHC, Phys. Lett. B 716 (2012) 1 [arXiv:1207.7214] [INSPIRE].

[4] V.A. Kuzmin, V.A. Rubakov and M.E. Shaposhnikov, On the anomalous electroweak baryon number nonconservation in the early universe, Phys. Lett. B 155 (1985) 36 [INSPIRE].

[5] M. Quirós, Field theory at finite temperature and phase transitions, Helv. Phys. Acta 67 (1994) 451 [INSPIRE].

[6] V.A. Rubakov and M.E. Shaposhnikov, Electroweak baryon number nonconservation in the early universe and in high-energy collisions, Usp. Fiz. Nauk 166 (1996) 493 [Phys. Usp. 39 (1996) 461] [hep-ph/9603208] [INSPIRE].

[7] K. Funakubo, CP violation and baryogenesis at the electroweak phase transition, Prog. Theor. Phys. 96 (1996) 475 [hep-ph/9608358] [InSPIRE].

[8] A. Riotto, Theories of baryogenesis, in ICTP summer school in high-energy physics and cosmology, (1998), pg. 326 [hep-ph/9807454] [INSPIRE].

[9] M. Trodden, Electroweak baryogenesis, Rev. Mod. Phys. 71 (1999) 1463 [hep-ph/9803479] [INSPIRE].

[10] W. Bernreuther, CP violation and baryogenesis, Lect. Notes Phys. 591 (2002) 237 [hep-ph/0205279] [INSPIRE].

[11] J.M. Cline, Baryogenesis, in Les Houches Summer School - session 86. Particle physics and cosmology: the fabric of spacetime, (2006) [hep-ph/0609145] [INSPIRE].

[12] D.E. Morrissey and M.J. Ramsey-Musolf, Electroweak baryogenesis, New J. Phys. 14 (2012) 125003 [arXiv: 1206.2942] [INSPIRE].

[13] T. Konstandin, Quantum transport and electroweak baryogenesis, Phys. Usp. 56 (2013) 747 [Usp. Fiz. Nauk 183 (2013) 785] [arXiv: 1302.6713] [INSPIRE].

[14] E. Senaha, Symmetry restoration and breaking at finite temperature: an introductory review, Symmetry 12 (2020) 733 [INSPIRE]. 
[15] A.I. Bochkarev, S.V. Kuzmin and M.E. Shaposhnikov, Electroweak baryogenesis and the Higgs boson mass problem, Phys. Lett. B 244 (1990) 275 [INSPIRE].

[16] K. Funakubo, A. Kakuto and K. Takenaga, The effective potential of electroweak theory with two massless Higgs doublets at finite temperature, Prog. Theor. Phys. 91 (1994) 341 [hep-ph/9310267] [INSPIRE].

[17] J.M. Cline and P.-A. Lemieux, Electroweak phase transition in two Higgs doublet models, Phys. Rev. D 55 (1997) 3873 [hep-ph/9609240] [INSPIRE].

[18] S. Kanemura, Y. Okada and E. Senaha, Electroweak baryogenesis and quantum corrections to the triple Higgs boson coupling, Phys. Lett. B 606 (2005) 361 [hep-ph/0411354] [INSPIRE].

[19] L. Fromme, S.J. Huber and M. Seniuch, Baryogenesis in the two-Higgs doublet model, JHEP 11 (2006) 038 [hep-ph/0605242] [INSPIRE].

[20] T.A. Chowdhury, M. Nemevšek, G. Senjanović and Y. Zhang, Dark matter as the trigger of strong electroweak phase transition, JCAP 02 (2012) 029 [arXiv:1110.5334] [INSPIRE].

[21] D. Borah and J.M. Cline, Inert doublet dark matter with strong electroweak phase transition, Phys. Rev. D 86 (2012) 055001 [arXiv:1204.4722] [INSPIRE].

[22] J.M. Cline and K. Kainulainen, Improved electroweak phase transition with subdominant inert doublet dark matter, Phys. Rev. D 87 (2013) 071701 [arXiv:1302.2614] [INSPIRE].

[23] G. Gil, P. Chankowski and M. Krawczyk, Inert dark matter and strong electroweak phase transition, Phys. Lett. B 717 (2012) 396 [arXiv:1207.0084] [INSPIRE].

[24] G.C. Dorsch, S.J. Huber and J.M. No, A strong electroweak phase transition in the 2HDM after LHC8, JHEP 10 (2013) 029 [arXiv:1305.6610] [INSPIRE].

[25] G.C. Dorsch, S.J. Huber, K. Mimasu and J.M. No, Echoes of the electroweak phase transition: discovering a second Higgs doublet through $A_{0} \rightarrow Z H_{0}$, Phys. Rev. Lett. 113 (2014) 211802 [arXiv:1405.5537] [inSPIRE].

[26] N. Blinov, S. Profumo and T. Stefaniak, The electroweak phase transition in the inert doublet model, JCAP 07 (2015) 028 [arXiv:1504.05949] [INSPIRE].

[27] K. Fuyuto and E. Senaha, Sphaleron and critical bubble in the scale invariant two Higgs doublet model, Phys. Lett. B 747 (2015) 152 [arXiv:1504.04291] [INSPIRE].

[28] K. Fuyuto, J. Hisano and E. Senaha, Toward verification of electroweak baryogenesis by electric dipole moments, Phys. Lett. B 755 (2016) 491 [arXiv:1510.04485] [INSPIRE].

[29] P. Basler, M. Mühlleitner and J. Wittbrodt, The CP-violating 2HDM in light of a strong first order electroweak phase transition and implications for Higgs pair production, JHEP 03 (2018) 061 [arXiv:1711.04097] [INSPIRE].

[30] K. Kainulainen, V. Keus, L. Niemi, K. Rummukainen, T.V.I. Tenkanen and V. Vaskonen, On the validity of perturbative studies of the electroweak phase transition in the two Higgs doublet model, JHEP 06 (2019) 075 [arXiv: 1904.01329] [INSPIRE].

[31] E. Senaha, Radiative corrections to triple Higgs coupling and electroweak phase transition: beyond one-loop analysis, Phys. Rev. D 100 (2019) 055034 [arXiv:1811.00336] [INSPIRE].

[32] F.P. Huang and E. Senaha, Enhanced $Z$ boson decays as a new probe of first-order electroweak phase transition at future lepton colliders, Phys. Rev. D 100 (2019) 035014 [arXiv: 1905.10283] [INSPIRE]. 
[33] X. Wang, F.P. Huang and X. Zhang, Gravitational wave and collider signals in complex two-Higgs doublet model with dynamical CP-violation at finite temperature, Phys. Rev. D 101 (2020) 015015 [arXiv: 1909.02978] [INSPIRE].

[34] M. Pietroni, The electroweak phase transition in a nonminimal supersymmetric model, Nucl. Phys. B 402 (1993) 27 [hep-ph/9207227] [INSPIRE].

[35] J.R. Espinosa, M. Quirós and F. Zwirner, On the electroweak phase transition in the minimal supersymmetric Standard Model, Phys. Lett. B 307 (1993) 106 [hep-ph/9303317] [INSPIRE].

[36] A. Brignole, J.R. Espinosa, M. Quirós and F. Zwirner, Aspects of the electroweak phase transition in the minimal supersymmetric Standard Model, Phys. Lett. B 324 (1994) 181 [hep-ph/9312296] [INSPIRE].

[37] A.T. Davies, C.D. Froggatt and R.G. Moorhouse, Electroweak baryogenesis in the next-to-minimal supersymmetric model, Phys. Lett. B 372 (1996) 88 [hep-ph/9603388] [INSPIRE].

[38] J.R. Espinosa, Dominant two loop corrections to the MSSM finite temperature effective potential, Nucl. Phys. B 475 (1996) 273 [hep-ph/9604320] [InSPIRE].

[39] B. de Carlos and J.R. Espinosa, The baryogenesis window in the MSSM, Nucl. Phys. B 503 (1997) 24 [hep-ph/9703212] [INSPIRE].

[40] S.J. Huber and M.G. Schmidt, Electroweak baryogenesis: concrete in a SUSY model with a gauge singlet, Nucl. Phys. B 606 (2001) 183 [hep-ph/0003122] [INSPIRE].

[41] K. Funakubo, S. Tao and F. Toyoda, CP violation in the Higgs sector and phase transition in the MSSM, Prog. Theor. Phys. 109 (2003) 415 [hep-ph/0211238] [INSPIRE].

[42] K. Funakubo, S. Tao and F. Toyoda, Phase transitions in the NMSSM, Prog. Theor. Phys. 114 (2005) 369 [hep-ph/0501052] [INSPIRE].

[43] S.J. Huber, T. Konstandin, T. Prokopec and M.G. Schmidt, Electroweak phase transition and baryogenesis in the NMSSM, Nucl. Phys. B 757 (2006) 172 [hep-ph/0606298] [INSPIRE].

[44] K. Funakubo and E. Senaha, Electroweak phase transition, critical bubbles and sphaleron decoupling condition in the MSSM, Phys. Rev. D 79 (2009) 115024 [arXiv:0905.2022] [INSPIRE].

[45] C.-W. Chiang and E. Senaha, Electroweak phase transitions in the secluded U(1)'-extended MSSM, JHEP 06 (2010) 030 [arXiv:0912.5069] [INSPIRE].

[46] D.J.H. Chung and A.J. Long, Electroweak phase transition in the $\mu \nu S S M$, Phys. Rev. D 81 (2010) 123531 [arXiv:1004.0942] [INSPIRE].

[47] S. Kanemura, E. Senaha and T. Shindou, First-order electroweak phase transition powered by additional F-term loop effects in an extended supersymmetric Higgs sector, Phys. Lett. B 706 (2011) 40 [arXiv:1109.5226] [InSPIRE].

[48] M. Carena, N.R. Shah and C.E.M. Wagner, Light dark matter and the electroweak phase transition in the NMSSM, Phys. Rev. D 85 (2012) 036003 [arXiv:1110.4378] [INSPIRE].

[49] K. Cheung, T.-J. Hou, J.S. Lee and E. Senaha, Singlino-driven electroweak baryogenesis in the next-to-MSSM, Phys. Lett. B 710 (2012) 188 [arXiv:1201.3781] [INSPIRE]. 
[50] R. Fok, G.D. Kribs, A. Martin and Y. Tsai, Electroweak baryogenesis in R-symmetric supersymmetry, Phys. Rev. D 87 (2013) 055018 [arXiv:1208.2784] [INSPIRE].

[51] S. Kanemura, E. Senaha, T. Shindou and T. Yamada, Electroweak phase transition and Higgs boson couplings in the model based on supersymmetric strong dynamics, JHEP 05 (2013) 066 [arXiv: 1211.5883] [INSPIRE].

[52] E. Senaha, $Z^{\prime}$-ino-driven electroweak baryogenesis in the UMSSM, Phys. Rev. D 88 (2013) 055014 [arXiv: 1308.3389] [INSPIRE].

[53] J. Kozaczuk, S. Profumo, L.S. Haskins and C.L. Wainwright, Cosmological phase transitions and their properties in the NMSSM, JHEP 01 (2015) 144 [arXiv:1407.4134] [INSPIRE].

[54] P. Athron, C. Balázs, A. Fowlie, G. Pozzo, G. White and Y. Zhang, Strong first-order phase transitions in the NMSSM - a comprehensive survey, JHEP 11 (2019) 151 [arXiv: 1908.11847] [INSPIRE].

[55] J. Choi and R.R. Volkas, Real Higgs singlet and the electroweak phase transition in the Standard Model, Phys. Lett. B 317 (1993) 385 [hep-ph/9308234] [INSPIRE].

[56] S.W. Ham, Y.S. Jeong and S.K. Oh, Electroweak phase transition in an extension of the Standard Model with a real Higgs singlet, J. Phys. G 31 (2005) 857 [hep-ph/0411352] [INSPIRE].

[57] A. Ahriche, What is the criterion for a strong first order electroweak phase transition in singlet models?, Phys. Rev. D 75 (2007) 083522 [hep-ph/0701192] [INSPIRE].

[58] S. Profumo, M.J. Ramsey-Musolf and G. Shaughnessy, Singlet Higgs phenomenology and the electroweak phase transition, JHEP 08 (2007) 010 [arXiv:0705.2425] [INSPIRE].

[59] J.R. Espinosa and M. Quirós, Novel effects in electroweak breaking from a hidden sector, Phys. Rev. D 76 (2007) 076004 [hep-ph/0701145] [INSPIRE].

[60] A. Ashoorioon and T. Konstandin, Strong electroweak phase transitions without collider traces, JHEP 07 (2009) 086 [arXiv:0904.0353] [INSPIRE].

[61] D.J.H. Chung and A.J. Long, Cosmological constant, dark matter and electroweak phase transition, Phys. Rev. D 84 (2011) 103513 [arXiv:1108.5193] [INSPIRE].

[62] J.R. Espinosa, T. Konstandin and F. Riva, Strong electroweak phase transitions in the Standard Model with a singlet, Nucl. Phys. B 854 (2012) 592 [arXiv:1107.5441] [InSPIRE].

[63] J.R. Espinosa, B. Gripaios, T. Konstandin and F. Riva, Electroweak baryogenesis in non-minimal composite Higgs models, JCAP 01 (2012) 012 [arXiv:1110.2876] [INSPIRE].

[64] J.M. Cline and K. Kainulainen, Electroweak baryogenesis and dark matter from a singlet Higgs, JCAP 01 (2013) 012 [arXiv:1210.4196] [INSPIRE].

[65] J.M. Cline, K. Kainulainen, P. Scott and C. Weniger, Update on scalar singlet dark matter, Phys. Rev. D 88 (2013) 055025 [Erratum ibid. 92 (2015) 039906] [arXiv:1306.4710] [INSPIRE].

[66] K. Fuyuto and E. Senaha, Improved sphaleron decoupling condition and the Higgs coupling constants in the real singlet-extended Standard Model, Phys. Rev. D 90 (2014) 015015 [arXiv: 1406.0433] [INSPIRE].

[67] T. Alanne, K. Tuominen and V. Vaskonen, Strong phase transition, dark matter and vacuum stability from simple hidden sectors, Nucl. Phys. B 889 (2014) 692 [arXiv: 1407.0688] [INSPIRE]. 
[68] S. Profumo, M.J. Ramsey-Musolf, C.L. Wainwright and P. Winslow, Singlet-catalyzed electroweak phase transitions and precision Higgs boson studies, Phys. Rev. D 91 (2015) 035018 [arXiv: 1407.5342] [INSPIRE].

[69] D. Curtin, P. Meade and C.-T. Yu, Testing electroweak baryogenesis with future colliders, JHEP 11 (2014) 127 [arXiv:1409.0005] [InSPIRE].

[70] J. Kozaczuk, Bubble expansion and the viability of singlet-driven electroweak baryogenesis, JHEP 10 (2015) 135 [arXiv:1506.04741] [INSPIRE].

[71] V. Vaskonen, Electroweak baryogenesis and gravitational waves from a real scalar singlet, Phys. Rev. D 95 (2017) 123515 [arXiv:1611.02073] [INSPIRE].

[72] D. Curtin, P. Meade and H. Ramani, Thermal resummation and phase transitions, Eur. Phys. J. C 78 (2018) 787 [arXiv: 1612.00466] [INSPIRE].

[73] G. Kurup and M. Perelstein, Dynamics of electroweak phase transition in singlet-scalar extension of the Standard Model, Phys. Rev. D 96 (2017) 015036 [arXiv:1704.03381] [INSPIRE].

[74] C.-W. Chiang, M.J. Ramsey-Musolf and E. Senaha, Standard Model with a complex scalar singlet: cosmological implications and theoretical considerations, Phys. Rev. D 97 (2018) 015005 [arXiv: 1707.09960] [INSPIRE].

[75] F.P. Huang, Z. Qian and M. Zhang, Exploring dynamical CP-violation induced baryogenesis by gravitational waves and colliders, Phys. Rev. D 98 (2018) 015014 [arXiv:1804.06813] [INSPIRE].

[76] C.-W. Chiang, Y.-T. Li and E. Senaha, Revisiting electroweak phase transition in the Standard Model with a real singlet scalar, Phys. Lett. B 789 (2019) 154 [arXiv: 1808.01098] [INSPIRE].

[77] A. Beniwal, M. Lewicki, M. White and A.G. Williams, Gravitational waves and electroweak baryogenesis in a global study of the extended scalar singlet model, JHEP 02 (2019) 183 [arXiv: 1810.02380] [INSPIRE].

[78] M. Chala, M. Ramos and M. Spannowsky, Gravitational wave and collider probes of a triplet Higgs sector with a low cutoff, Eur. Phys. J. C 79 (2019) 156 [arXiv:1812.01901] [INSPIRE].

[79] S. Tulin and P. Winslow, Anomalous B meson mixing and baryogenesis, Phys. Rev. D 84 (2011) 034013 [arXiv:1105.2848] [INSPIRE].

[80] J.M. Cline, K. Kainulainen and M. Trott, Electroweak baryogenesis in two Higgs doublet models and B meson anomalies, JHEP 11 (2011) 089 [arXiv:1107.3559] [INSPIRE].

[81] T. Liu, M.J. Ramsey-Musolf and J. Shu, Electroweak beautygenesis: from $b \rightarrow s$ CP-violation to the cosmic baryon asymmetry, Phys. Rev. Lett. 108 (2012) 221301 [arXiv:1109.4145] [INSPIRE].

[82] C.-W. Chiang, K. Fuyuto and E. Senaha, Electroweak baryogenesis with lepton flavor violation, Phys. Lett. B 762 (2016) 315 [arXiv:1607.07316] [INSPIRE].

[83] H.-K. Guo, Y.-Y. Li, T. Liu, M. Ramsey-Musolf and J. Shu, Lepton-flavored electroweak baryogenesis, Phys. Rev. D 96 (2017) 115034 [arXiv:1609.09849] [INSPIRE].

[84] K. Fuyuto, W.-S. Hou and E. Senaha, Electroweak baryogenesis driven by extra top Yukawa couplings, Phys. Lett. B 776 (2018) 402 [arXiv:1705.05034] [INSPIRE]. 
[85] T. Modak and E. Senaha, Electroweak baryogenesis via bottom transport, Phys. Rev. D 99 (2019) 115022 [arXiv: 1811.08088] [inSPIRE].

[86] K. Fuyuto, W.-S. Hou and E. Senaha, Cancellation mechanism for the electron electric dipole moment connected with the baryon asymmetry of the universe, Phys. Rev. D 101 (2020) 011901 [arXiv: 1910.12404] [INSPIRE].

[87] K. Ishikawa, T. Kitahara and M. Takimoto, Towards a scale free electroweak baryogenesis, Phys. Rev. D 91 (2015) 055004 [arXiv:1410.5432] [INSPIRE].

[88] I. Baldes and G. Servant, High scale electroweak phase transition: baryogenesis \& symmetry non-restoration, JHEP 10 (2018) 053 [arXiv: 1807.08770] [INSPIRE].

[89] O. Matsedonskyi and G. Servant, High-temperature electroweak symmetry non-restoration from new fermions and implications for baryogenesis, JHEP 09 (2020) 012 [arXiv: 2002.05174] [INSPIRE].

[90] A. Djouadi, The anatomy of electro-weak symmetry breaking. I: the Higgs boson in the Standard Model, Phys. Rept. 457 (2008) 1 [hep-ph/0503172] [INSPIRE].

[91] G.C. Branco, P.M. Ferreira, L. Lavoura, M.N. Rebelo, M. Sher and J.P. Silva, Theory and phenomenology of two-Higgs-doublet models, Phys. Rept. 516 (2012) 1 [arXiv:1106.0034] [INSPIRE].

[92] N. Cabibbo, Unitary symmetry and leptonic decays, Phys. Rev. Lett. 10 (1963) 531 [INSPIRE].

[93] M. Kohda, T. Modak and W.-S. Hou, Searching for new scalar bosons via triple-top signature in $c g \rightarrow t S^{0} \rightarrow t t \bar{t}$, Phys. Lett. B 776 (2018) 379 [arXiv:1710.07260] [INSPIRE].

[94] T. Modak, Probing an additional bottom Yukawa coupling via bg $\rightarrow b A \rightarrow b Z H$ signature, Phys. Rev. D 100 (2019) 035018 [arXiv: 1905.02137] [inSPIRE].

[95] S. Davidson and H.E. Haber, Basis-independent methods for the two-Higgs-doublet model, Phys. Rev. D 72 (2005) 035004 [Erratum ibid. 72 (2005) 099902] [hep-ph/0504050] [INSPIRE].

[96] W.-S. Hou and M. Kikuchi, Approximate alignment in two Higgs doublet model with extra Yukawa couplings, EPL 123 (2018) 11001 [arXiv:1706.07694] [INSPIRE].

[97] ATLAS collaboration, Search for heavy resonances decaying into a $W$ or $Z$ boson and a Higgs boson in final states with leptons and b-jets in $36 \mathrm{fb}^{-1}$ of $\sqrt{s}=13 \mathrm{TeV}$ pp collisions with the ATLAS detector, JHEP 03 (2018) 174 [Erratum ibid. 11 (2018) 051] [arXiv: 1712.06518] [INSPIRE].

[98] CMS collaboration, Search for a heavy pseudoscalar boson decaying to a $Z$ and a Higgs boson at $\sqrt{s}=13 \mathrm{TeV}$, Eur. Phys. J. C 79 (2019) 564 [arXiv: 1903.00941] [InSPIRE].

[99] P.M. Ferreira, S. Liebler and J. Wittbrodt, $p p \rightarrow A \rightarrow Z h$ and the wrong-sign limit of the two-Higgs-doublet model, Phys. Rev. D 97 (2018) 055008 [arXiv:1711.00024] [INSPIRE].

[100] N.M. Coyle, B. Li and C.E.M. Wagner, Wrong sign bottom Yukawa coupling in low energy supersymmetry, Phys. Rev. D 97 (2018) 115028 [arXiv:1802.09122] [INSPIRE].

[101] A. Djouadi, L. Maiani, A. Polosa, J. Quevillon and V. Riquer, Fully covering the MSSM Higgs sector at the LHC, JHEP 06 (2015) 168 [arXiv:1502.05653] [INSPIRE].

[102] N. Craig, F. D'Eramo, P. Draper, S. Thomas and H. Zhang, The hunt for the rest of the Higgs bosons, JHEP 06 (2015) 137 [arXiv:1504.04630] [INSPIRE]. 
[103] J. Hajer, Y.-Y. Li, T. Liu and J.F.H. Shiu, Heavy Higgs bosons at $14 \mathrm{TeV}$ and $100 \mathrm{TeV}$, JHEP 11 (2015) 124 [arXiv: 1504.07617] [INSPIRE].

[104] E. Alvarez and M. Estevez, têtb $\bar{b}$ as a probe of new physics at the LHC, Phys. Rev. D 96 (2017) 035016 [arXiv: 1701.04427] [INSPIRE].

[105] S. Gori, I.-W. Kim, N.R. Shah and K.M. Zurek, Closing the wedge: search strategies for extended Higgs sectors with heavy flavor final states, Phys. Rev. D 93 (2016) 075038 [arXiv: 1602.02782] [INSPIRE].

[106] ATLAS collaboration, Search for heavy Higgs bosons A/H decaying to a top quark pair in pp collisions at $\sqrt{s}=8 \mathrm{TeV}$ with the ATLAS detector, Phys. Rev. Lett. 119 (2017) 191803 [arXiv: 1707.06025] [INSPIRE].

[107] CMS collaboration, Search for heavy Higgs bosons decaying to a top quark pair in proton-proton collisions at $\sqrt{s}=13 \mathrm{TeV}$, JHEP 04 (2020) 171 [arXiv: 1908.01115] [INSPIRE].

[108] M. Carena and Z. Liu, Challenges and opportunities for heavy scalar searches in the $t \bar{t}$ channel at the LHC, JHEP 11 (2016) 159 [arXiv:1608.07282] [INSPIRE].

[109] S. Kanemura, H. Yokoya and Y.-J. Zheng, Searches for additional Higgs bosons in multi-top-quarks events at the LHC and the International Linear Collider, Nucl. Phys. B 898 (2015) 286 [arXiv: 1505.01089] [INSPIRE].

[110] N. Craig, J. Hajer, Y.-Y. Li, T. Liu and H. Zhang, Heavy Higgs bosons at low $\tan \beta$ : from the LHC to $100 \mathrm{TeV}$, JHEP 01 (2017) 018 [arXiv:1605.08744] [INSPIRE].

[111] W.-S. Hou, M. Kohda and T. Modak, Implications of four-top and top-pair studies on triple-top production, Phys. Lett. B 798 (2019) 134953 [arXiv:1906.09703] [INSPIRE].

[112] D. Eriksson, J. Rathsman and O. Stal, 2HDMC: two-Higgs-doublet model calculator physics and manual, Comput. Phys. Commun. 181 (2010) 189 [arXiv:0902.0851] [INSPIRE].

[113] M.E. Peskin and T. Takeuchi, Estimation of oblique electroweak corrections, Phys. Rev. D 46 (1992) 381 [INSPIRE].

[114] C.D. Froggatt, R.G. Moorhouse and I.G. Knowles, Leading radiative corrections in two scalar doublet models, Phys. Rev. D 45 (1992) 2471 [INSPIRE].

[115] H.E. Haber and O. Stål, New LHC benchmarks for the CP-conserving two-Higgs-doublet model, Eur. Phys. J. C 75 (2015) 491 [Erratum ibid. 76 (2016) 312] [arXiv:1507.04281] [INSPIRE].

[116] GFitTer Group collaboration, The global electroweak fit at NNLO and prospects for the LHC and ILC, Eur. Phys. J. C $\mathbf{7 4}$ (2014) 3046 [arXiv:1407.3792] [InSPIRE].

[117] Gfitter website, http://project-gfitter.web.cern.ch/project-gfitter/Oblique_Parameters/.

[118] W.-S. Hou, M. Kohda and T. Modak, Top-assisted di-Higgs boson production motivated by baryogenesis, Phys. Rev. D 99 (2019) 055046 [arXiv: 1901.00105] [InSPIRE].

[119] W.-S. Hou and T. Modak, Prospects for $t Z H$ and $t Z h$ production at the LHC, Phys. Rev. D 101 (2020) 035007 [arXiv: 1911.06010] [INSPIRE].

[120] M. Ciuchini, G. Degrassi, P. Gambino and G.F. Giudice, Next-to-leading QCD corrections to $B \rightarrow X_{s} \gamma$ : Standard Model and two Higgs doublet model, Nucl. Phys. B 527 (1998) 21 [hep-ph/9710335] [INSPIRE]. 
[121] K.G. Chetyrkin, M. Misiak and M. Münz, Weak radiative $B$ meson decay beyond leading logarithms, Phys. Lett. B 400 (1997) 206 [Erratum ibid. 425 (1998) 414] [hep-ph/9612313] [INSPIRE].

[122] B. Altunkaynak, W.-S. Hou, C. Kao, M. Kohda and B. McCoy, Flavor changing heavy Higgs interactions at the LHC, Phys. Lett. B 751 (2015) 135 [arXiv:1506.00651] [INSPIRE].

[123] HFLAV collaboration, Averages of b-hadron, c-hadron, and $\tau$-lepton properties as of summer 2016, Eur. Phys. J. C 77 (2017) 895 [arXiv:1612.07233] [INSPIRE].

[124] M. Czakon, P. Fiedler, T. Huber, M. Misiak, T. Schutzmeier and M. Steinhauser, The $\left(Q_{7}, Q_{1,2}\right)$ contribution to $\bar{B} \rightarrow X_{s} \gamma$ at $O\left(\alpha_{\mathrm{s}}^{2}\right)$, JHEP 04 (2015) 168 [arXiv:1503.01791] [INSPIRE].

[125] A. Crivellin, A. Kokulu and C. Greub, Flavor-phenomenology of two-Higgs-doublet models with generic Yukawa structure, Phys. Rev. D 87 (2013) 094031 [arXiv:1303.5877] [INSPIRE].

[126] C.-Q. Geng and J.-N. Ng, Charged Higgs effect in $B_{d}^{0}-\bar{B}_{d}^{0}$ mixing, $K \rightarrow \pi \nu \bar{\nu}$ decay and rare decays of B mesons, Phys. Rev. D 38 (1988) 2857 [Erratum ibid. 41 (1990) 1715] [INSPIRE].

[127] UTFIT collaboration, Constraints on new physics from the quark mixing unitarity triangle, Phys. Rev. Lett. 97 (2006) 151803 [hep-ph/0605213] [INSPIRE].

[128] New physics fit results: summer 2018 webpage, http://www.utfit.org/UTfit/ResultsSummer2018NP.

[129] A.L. Kagan and M. Neubert, Direct CP-violation in $B \rightarrow X_{s} \gamma$ decays as a signature of new physics, Phys. Rev. D 58 (1998) 094012 [hep-ph/9803368] [INSPIRE].

[130] M. Benzke, S.J. Lee, M. Neubert and G. Paz, Long-distance dominance of the CP asymmetry in $B \rightarrow X_{s, d}+\gamma$ decays, Phys. Rev. Lett. 106 (2011) 141801 [arXiv:1012.3167] [INSPIRE].

[131] BELLE collaboration, Measurements of isospin asymmetry and difference of direct $C P$ asymmetries in inclusive $B \rightarrow X_{s} \gamma$ decays, Phys. Rev. D 99 (2019) 032012 [arXiv: 1807.04236] [INSPIRE].

[132] K. Fuyuto, M. Ramsey-Musolf and T. Shen, Electric dipole moments from CP-violating scalar leptoquark interactions, Phys. Lett. B 788 (2019) 52 [arXiv:1804.01137] [InSPIRE].

[133] T. Abe, J. Hisano, T. Kitahara and K. Tobioka, Gauge invariant Barr-Zee type contributions to fermionic EDMs in the two-Higgs doublet models, JHEP 01 (2014) 106 [Erratum ibid. 04 (2016) 161] [arXiv:1311.4704] [INSPIRE].

[134] D. Bowser-Chao, D. Chang and W.-Y. Keung, Electron electric dipole moment from CP-violation in the charged Higgs sector, Phys. Rev. Lett. 79 (1997) 1988 [hep-ph/9703435] [INSPIRE].

[135] M. Kobayashi and T. Maskawa, CP violation in the renormalizable theory of weak interaction, Prog. Theor. Phys. 49 (1973) 652 [InSPIRE].

[136] W. Dekens, J. de Vries, M. Jung and K.K. Vos, The phenomenology of electric dipole moments in models of scalar leptoquarks, JHEP 01 (2019) 069 [arXiv:1809.09114] [INSPIRE].

[137] NEDM collaboration, Measurement of the permanent electric dipole moment of the neutron, Phys. Rev. Lett. 124 (2020) 081803 [arXiv:2001.11966] [INSPIRE]. 
[138] B. Graner, Y. Chen, E.G. Lindahl and B.R. Heckel, Reduced limit on the permanent electric dipole moment of ${ }^{199} \mathrm{Hg}$, Phys. Rev. Lett. 116 (2016) 161601 [Erratum ibid. 119 (2017) 119901] [arXiv: 1601.04339] [INSPIRE].

[139] J. Hisano, D. Kobayashi, W. Kuramoto and T. Kuwahara, Nucleon electric dipole moments in high-scale supersymmetric models, JHEP 11 (2015) 085 [arXiv: 1507.05836] [INSPIRE].

[140] J.R. Ellis, J.S. Lee and A. Pilaftsis, Electric dipole moments in the MSSM reloaded, JHEP 10 (2008) 049 [arXiv: 0808.1819] [INSPIRE].

[141] J. Ellis, J.S. Lee and A. Pilaftsis, Maximal electric dipole moments of nuclei with enhanced Schiff moments, JHEP 02 (2011) 045 [arXiv:1101.3529] [INSPIRE].

[142] K. Cheung, J.S. Lee, E. Senaha and P.-Y. Tseng, Confronting higgcision with electric dipole moments, JHEP 06 (2014) 149 [arXiv: 1403.4775] [INSPIRE].

[143] S. Haciömeroğlu and Y.K. Semertzidis, Hybrid ring design in the storage-ring proton electric dipole moment experiment, Phys. Rev. Accel. Beams 22 (2019) 034001 [arXiv: 1806.09319] [INSPIRE].

[144] V. Anastassopoulos et al., A storage ring experiment to detect a proton electric dipole moment, Rev. Sci. Instrum. 87 (2016) 115116 [arXiv:1502.04317] [INSPIRE].

[145] CMS collaboration, Search for beyond the Standard Model Higgs bosons decaying into a $b \bar{b}$ pair in pp collisions at $\sqrt{s}=13 \mathrm{TeV}$, JHEP 08 (2018) 113 [arXiv:1805.12191] [INSPIRE].

[146] ATLAS collaboration, Search for heavy neutral Higgs bosons produced in association with b-quarks and decaying to b-quarks at $\sqrt{s}=13 \mathrm{TeV}$ with the ATLAS detector, Tech. Rep. ATLAS-CONF-2019-010, CERN, Geneva, Switzerland (2019).

[147] ATLAS collaboration, Search for charged Higgs bosons decaying into top and bottom quarks at $\sqrt{s}=13 \mathrm{TeV}$ with the ATLAS detector, JHEP 11 (2018) 085 [arXiv:1808.03599] [INSPIRE].

[148] CMS collaboration, Search for a charged Higgs boson decaying into top and bottom quarks in events with electrons or muons in proton-proton collisions at $\sqrt{s}=13 \mathrm{TeV}$, JHEP 01 (2020) 096 [arXiv: 1908.09206] [INSPIRE].

[149] CMS collaboration, Search for charged Higgs bosons decaying into a top and a bottom quark in the all-jet final state of pp collisions at $\sqrt{s}=13 \mathrm{TeV}$, JHEP 07 (2020) 126 [arXiv: 2001. 07763] [INSPIRE].

[150] J. Alwall et al., The automated computation of tree-level and next-to-leading order differential cross sections, and their matching to parton shower simulations, JHEP 07 (2014) 079 [arXiv: 1405.0301] [INSPIRE].

[151] NNPDF collaboration, Parton distributions with QED corrections, Nucl. Phys. B 877 (2013) 290 [arXiv: 1308.0598] [INSPIRE].

[152] A. Alloul, N.D. Christensen, C. Degrande, C. Duhr and B. Fuks, FeynRules $2.0-a$ complete toolbox for tree-level phenomenology, Comput. Phys. Commun. 185 (2014) 2250 [arXiv: 1310.1921] [INSPIRE].

[153] CMS collaboration, Search for production of four top quarks in final states with same-sign or multiple leptons in proton-proton collisions at $\sqrt{s}=13 \mathrm{TeV}$, Eur. Phys. J. C 80 (2020) 75 [arXiv: 1908.06463] [INSPIRE]. 
[154] T. Sjöstrand, S. Mrenna and P.Z. Skands, PYTHIA 6.4 physics and manual, JHEP 05 (2006) 026 [hep-ph/0603175] [INSPIRE].

[155] DELPHES 3 collaboration, DELPHES 3, a modular framework for fast simulation of a generic collider experiment, JHEP 02 (2014) 057 [arXiv:1307.6346] [INSPIRE].

[156] M.L. Mangano, M. Moretti, F. Piccinini and M. Treccani, Matching matrix elements and shower evolution for top-quark production in hadronic collisions, JHEP 01 (2007) 013 [hep-ph/0611129] [INSPIRE].

[157] J. Alwall et al., Comparative study of various algorithms for the merging of parton showers and matrix elements in hadronic collisions, Eur. Phys. J. C 53 (2008) 473 [arXiv:0706.2569] [INSPIRE].

[158] ATLAS-CMS recommended $t \bar{t}$ cross section predictions webpage, https://twiki.cern.ch/twiki/bin/view/LHCPhysics/TtbarNNLO.

[159] N. Kidonakis, Two-loop soft anomalous dimensions for single top quark associated production with a $W^{-}$or $H^{-}$, Phys. Rev. D 82 (2010) 054018 [arXiv:1005.4451] [INSPIRE].

[160] Y. Li and F. Petriello, Combining QCD and electroweak corrections to dilepton production in FEWZ, Phys. Rev. D 86 (2012) 094034 [arXiv:1208.5967] [INSPIRE].

[161] W.-S. Hou, M. Kohda and T. Modak, Search for $t Z^{\prime}$ associated production induced by $t c Z^{\prime}$ couplings at the LHC, Phys. Rev. D 96 (2017) 015037 [arXiv:1702.07275] [INSPIRE].

[162] J. Campbell, R.K. Ellis and R. Röntsch, Single top production in association with a $Z$ boson at the LHC, Phys. Rev. D 87 (2013) 114006 [arXiv:1302.3856] [INSPIRE].

[163] SM Higgs production cross sections at $\sqrt{s}=14 \mathrm{TeV}$ webpage, https://twiki.cern.ch/twiki/bin/view/LHCPhysics/CERNYellowReportPageAt14TeV2010.

[164] J.M. Campbell and R. Ellis, $t \bar{t} W^{ \pm}$production and decay at NLO, JHEP 07 (2012) 052 [arXiv: 1204.5678] [INSPIRE].

[165] M. Grazzini, S. Kallweit, D. Rathlev and M. Wiesemann, $W^{ \pm} Z$ production at hadron colliders in NNLO QCD, Phys. Lett. B 761 (2016) 179 [arXiv:1604.08576] [INSPIRE].

[166] ATLAS-CMS recommended predictions for single-top cross sections using the Hathor v2.1 program webpage, https://twiki.cern.ch/twiki/bin/view/LHCPhysics/SingleTopRefXsec.

[167] G. Cowan, K. Cranmer, E. Gross and O. Vitells, Asymptotic formulae for likelihood-based tests of new physics, Eur. Phys. J. C 71 (2011) 1554 [Erratum ibid. 73 (2013) 2501] [arXiv: 1007.1727] [INSPIRE].

[168] W.-S. Hou, M. Kohda and T. Modak, Constraining a lighter exotic scalar via same-sign top, Phys. Lett. B 786 (2018) 212 [arXiv:1808.00333] [INSPIRE].

[169] W.-S. Hou, G.-L. Lin, C.-Y. Ma and C.P. Yuan, Probing flavor changing neutral Higgs couplings at LHC, Phys. Lett. B 409 (1997) 344 [hep-ph/9702260] [InSPIRE].

[170] S. Iguro and K. Tobe, $R\left(D^{(*)}\right)$ in a general two Higgs doublet model, Nucl. Phys. B 925 (2017) 560 [arXiv: 1708.06176] [INSPIRE].

[171] W. Altmannshofer, J. Eby, S. Gori, M. Lotito, M. Martone and D. Tuckler, Collider signatures of flavorful Higgs bosons, Phys. Rev. D 94 (2016) 115032 [arXiv:1610.02398] [INSPIRE]. 
[172] W. Altmannshofer, B. Maddock and D. Tuckler, Rare top decays as probes of flavorful Higgs bosons, Phys. Rev. D 100 (2019) 015003 [arXiv: 1904.10956] [INSPIRE].

[173] S. Gori, C. Grojean, A. Juste and A. Paul, Heavy Higgs searches: flavour matters, JHEP 01 (2018) 108 [arXiv: 1710.03752] [INSPIRE].

[174] W.-S. Hou, M. Kohda and T. Modak, Probing for extra top Yukawa couplings in light of $t \bar{t} h(125)$ observation, Phys. Rev. D 98 (2018) 075007 [arXiv: 1806.06018] [INSPIRE].

[175] BeLLE-II collaboration, The Belle II physics book, PTEP 2019 (2019) 123C01 [Erratum ibid. 2020 (2020) 029201] [arXiv: 1808.10567] [INSPIRE].

[176] C.-H. Chen and T. Nomura, Charged Higgs boson contribution to $B_{q}^{-} \rightarrow \ell \bar{\nu}$ and $\bar{B} \rightarrow(P, V) \ell \bar{\nu}$ in a generic two-Higgs doublet model, Phys. Rev. D 98 (2018) 095007 [arXiv: 1803.00171] [INSPIRE].

[177] W.-S. Hou, M. Kohda and T. Modak, Unraveling the couplings of a Drell-Yan produced $Z^{\prime}$ with heavy-flavor tagging, Phys. Rev. D 98 (2018) 015002 [arXiv:1801.02579] [INSPIRE].

[178] J.M. Campbell, R. Ellis, F. Maltoni and S. Willenbrock, Higgs-boson production in association with a single bottom quark, Phys. Rev. D 67 (2003) 095002 [hep-ph/0204093] [INSPIRE].

[179] D. Dicus, T. Stelzer, Z. Sullivan and S. Willenbrock, Higgs boson production in association with bottom quarks at next-to-leading order, Phys. Rev. D 59 (1999) 094016 [hep-ph/9811492] [INSPIRE].

[180] F. Maltoni, T. McElmurry and S. Willenbrock, Inclusive production of a Higgs or $Z$ boson in association with heavy quarks, Phys. Rev. D 72 (2005) 074024 [hep-ph/0505014] [INSPIRE].

[181] R.V. Harlander and W.B. Kilgore, Higgs boson production in bottom quark fusion at next-to-next-to leading order, Phys. Rev. D 68 (2003) 013001 [hep-ph/0304035] [INSPIRE].

[182] F. Maltoni, Z. Sullivan and S. Willenbrock, Higgs-boson production via bottom-quark fusion, Phys. Rev. D 67 (2003) 093005 [hep-ph/0301033] [InSPIRE].

[183] J. Pumplin, D.R. Stump, J. Huston, H.L. Lai, P.M. Nadolsky and W.K. Tung, New generation of parton distributions with uncertainties from global QCD analysis, JHEP 07 (2002) 012 [hep-ph/0201195] [INSPIRE].

[184] F. Maltoni, G. Ridolfi and M. Ubiali, b-initiated processes at the LHC: a reappraisal, JHEP 07 (2012) 022 [Erratum ibid. 04 (2013) 095] [arXiv: 1203.6393] [INSPIRE].

[185] J. Butterworth et al., PDF4LHC recommendations for LHC run II, J. Phys. G 43 (2016) 023001 [arXiv: 1510.03865] [INSPIRE]. 